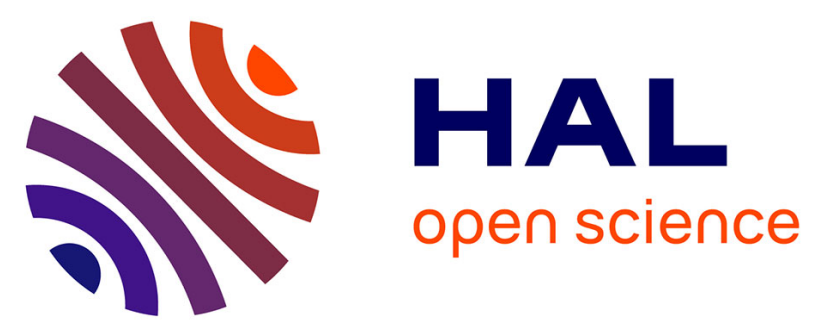

\title{
Equilibrated warping: Finite element image registration with finite strain equilibrium gap regularization
}

\author{
Martin Genet, C. T Stoeck, C. von Deuster, L. C. Lee, S. Kozerke
}

\section{To cite this version:}

Martin Genet, C. T Stoeck, C. von Deuster, L. C. Lee, S. Kozerke. Equilibrated warping: Finite element image registration with finite strain equilibrium gap regularization. Medical Image Analysis, 2018, 50, pp.1-22. 10.1016/j.media.2018.07.007 . hal-01882366

\section{HAL Id: hal-01882366 https://hal.science/hal-01882366}

Submitted on 27 Sep 2018

HAL is a multi-disciplinary open access archive for the deposit and dissemination of scientific research documents, whether they are published or not. The documents may come from teaching and research institutions in France or abroad, or from public or private research centers.
L'archive ouverte pluridisciplinaire HAL, est destinée au dépôt et à la diffusion de documents scientifiques de niveau recherche, publiés ou non, émanant des établissements d'enseignement et de recherche français ou étrangers, des laboratoires publics ou privés. 


\title{
Equilibrated Warping: Finite Element Image Registration with Finite Strain Equilibrium Gap Regularization
}

 \\ ${ }^{a}$ Laboratoire de Mécanique des Solides, École Polytechnique / C.N.R.S. / Université Paris-Saclay, \\ Palaiseau, France \\ ${ }^{b}$ M3DISIM team, Inria / Université Paris-Saclay, Palaiseau, France \\ ${ }^{c}$ Institute for Biomedical Engineering, University and ETH Zurich, Switzerland \\ ${ }^{d}$ Department of Mechanical Engineering, Michigan State University, East Lansing, USA
}

\begin{abstract}
In this paper, we propose a novel continuum finite strain formulation of the equilibrium gap regularization for image registration. The equilibrium gap regularization essentially penalizes any deviation from the solution of a hyperelastic body in equilibrium with arbitrary loads prescribed at the boundary. It thus represents a regularization with strong mechanical basis, especially suited for cardiac image analysis. We describe the consistent linearization and discretization of the regularized image registration problem, in the framework of the finite elements method. The method is implemented using FEniCS \& VTK, and distributed as a freely available python library. We show that the equilibrated warping method is effective and robust: regularization strength and image noise have minimal impact on motion tracking, especially when compared to strain-based regularization methods such as hyperelastic warping. We also show that equilibrated warping is able to extract main deformation features on both tagged and untagged cardiac magnetic resonance images.
\end{abstract}

Highlights.

- Continuum finite strain formulation of the equilibrium gap regularization principle

- Consistent linearization and finite element discretization

- Efficient implementation based on FEniCS \& VTK, freely available as a python library

- Equilibrium gap regularization allows the registration of highly noisy images

- Equilibrated warping can extract strains from cardiac magnetic resonance images

Keywords:

Finite elements, Image registration, Equilibrium gap regularization, Cardiac magnetic resonance imaging

${ }^{*}$ Corresponding author. martin.genet@polytechnique.edu 


\section{Contents}

1 Introduction $\quad 2$

2 Methods $\quad 4$

2.1 Finite element-based image registration . . . . . . . . . . . . . . 4

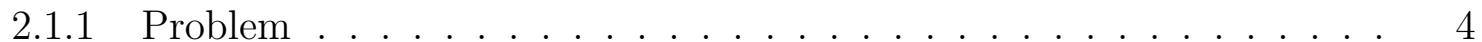

2.1 .2 Resolution . . . . . . . . . . . . . . . . . 6

2.1 .3 Implementation . . . . . . . . . . . . . . . . . . . . . . 9

2.2 Image synthesis, acquisition, combination and segmentation . . . . . . . . 10

2.2 .1 Image synthesis . . . . . . . . . . . . . . . . . . . . . 10

2.2 .2 Image acquisition and combination . . . . . . . . . . . . . 11

2.2 .3 Image segmentation . . . . . . . . . . . . . . . . . . . . . 12

3 Results \& Discussion 13

3.1 Validation on in silico images . . . . . . . . . . . . . . . . . . . . . 13

3.1.1 Influence of regularization strength . . . . . . . . . . . . . . 13

3.1 .2 Influence of mesh density . . . . . . . . . . . . . . . . . . . 16

3.2 Results on in vivo images . . . . . . . . . . . . . . . . . . 16

3.2.1 Results on in vivo tagged images . . . . . . . . . . . . . . . 16

3.2.2 Results on in vivo untagged images . . . . . . . . . . . . . . . . . 18

3.3 Limitations . . . . . . . . . . . . . . . . . . . . . . . . . . 24

\section{Conclusion $\quad 24$}

$\begin{array}{llll}\text { Appendix A } & \text { Validation using Motion Tracking Challenge } & 28\end{array}$

$\begin{array}{llll}\text { Appendix B Vitae } & 28\end{array}$

Appendix B.1 Martin Genet . . . . . . . . . . . . . . . . . . 28

Appendix B.2 Christian Stoeck . . . . . . . . . . . . . . . . . 29

Appendix B.3 Constantin von Deuster . . . . . . . . . . . . . . . . 29

Appendix B.4 Lik Chuan Lee . . . . . . . . . . . . . . . . . . . . . . 29

Appendix B.5 Sebastian Kozerke . . . . . . . . . . . . . . . . . . 29

$\begin{array}{ll}\text { References } & 30\end{array}$

\section{Introduction}

Image processing, in particular image registration for motion tracking, is playing an important role in biomedical imaging [Tobon-Gomez et al., 2013; Sotiras et al., 2013] and in other domains such as materials and mechanical engineering [Sutton and Hild, 2015]. However, despite important progress made in the past decades, robustness, efficiency and precision of the existing methods must still be improved to translate them into medical 
and engineering applications. In this paper we propose a novel regularization approach that has a strong mechanical basis, and apply it to finite element-based image registration problems. We illustrate our approach on cardiac motion tracking from magnetic resonance (MR) images.

MR imaging (MRI) is a powerful tool that can be used to quantify the motion of the beating heart in vivo and non-invasively, with wide ranging clinical applications. They include the diagnosis of coronary artery diseases, myocardial ischemia and infarction, non-ischemic cardiomyopathies, ventricular dyssynchrony, etc. [Shehata et al., 2009; Ibrahim, 2011]. Besides diagnosing heart diseases, cardiac motion tracking is also used as a component of other MRI techniques, for instance in in vivo diffusion tensor imaging [Stoeck et al., 2015; von Deuster et al., 2015], which has high clinical relevance. In the field of personalized computational modeling [Krishnamurthy et al., 2013; Lee et al., 2014], cardiac motion tracking is used to estimate model parameters that could serve as biomarkers for cardiovascular diseases [Sermesant et al., 2006; Imperiale et al., 2011].

Since regular anatomical cine MR images have little contrast within the myocardial wall, tagged MRI was designed to track material points through the generation and imaging of a magnetization grid (SPAtial Modulation of Magnetization, SPAMM) [Zerhouni et al., 1988; Axel and Dougherty, 1989]. It was later improved with the Complementary SPAMM (CSPAMM) method, which prevents tag fading [Fischer et al., 1993]. Accelerated wholeheart 3D sequences (3D CSPAMM) have since been proposed [Ryf et al., 2002], which mitigate misregistration issues common with multi-slice acquisitions and allow for fast and reliable tracking of the entire left ventricle throughout the cardiac cycle (except for enddiastole) in only three [Rutz et al., 2008], or even a single [Stoeck et al., 2012] breath hold.

Multiple approaches have been proposed to post-process cardiac magnetic resonance images and extract motion and deformation fields [Wang and Amini, 2012; Tobon-Gomez et al., 2013]. They vary in the nature of the a priori knowledge that is used to better distinguish signal from noise, and how it is incorporated. A first distinction exists between data assimilation and image registration techniques. In data assimilation, a realistic physical model is used and its parameters are estimated to best match the acquired images [Sermesant et al., 2006; Sainte-Marie et al., 2006]. This estimation is either variational [Delingette et al., 2012] or sequential [Moireau et al., 2008; Chapelle et al., 2013]. In other communities, this is called integrated image correlation [Hild and Roux, 2006; Hild et al., 2016]. Conversely, in image registration techniques, which are the focus of this paper, only limited a priori knowledge is required, solely to regularize the registration problem. The strengths and weaknesses of both approaches are opposite: using a realistic model as regularizer allows to process low resolution and/or noisy data while extracting meaningful physical parameters; on the other hand the processing is highly dependent on the validity of the model.

Within image registration methods, another distinction exists, between Fourier-based and tracking-based methods [Tobon-Gomez et al., 2013]. Among Fourier-based methods, HARmonic Phases (HARP)-based techniques [Osman et al., 1999; Garot et al., 2000] are the most commonly used methods, which have enabled fully automatic post-processing of tagged images. Based on similar concepts, the SinMod method [Arts et al., 2010; Wang 
et al., 2013] offers improved motion tracking performance. However, these methods are limited to tagged images, as HARP requires phase data, and SinMod makes use of the sine modulation of magnitude data. In contrast, tracking-based methods, which are the focus of this paper, can be applied to any type of images.

A final notable distinction exists, within tracking-based image registration techniques, between local and global approaches [Hild and Roux, 2012]. In local approaches, images are correlated region by region [Lenoir et al., 2007], while in global approaches they are correlated at once [Veress et al., 2005; Phatak et al., 2009]. Note that hybrid methods have been proposed, which efficiently alternate between local and global steps [Thirion, 1998; Vercauteren et al., 2008].

The present paper focuses on global tracking-based image registration, specifically on the regularization of the registration problem. Basic mathematical regularization such as Laplacian smoothing [Passieux and Périé, 2012] penalizes rigid body rotations and are thus limited to very small deformations. Fluid-like mechanical regularization has been proposed in [Christensen et al., 1996], which is not suitable for the (solid) myocardium as it does not enforce the continuity of the displacement field. Incompressibility has also been used as a regularizer [Mansi et al., 2011; McLeod et al., 2012], but it represents a strong kinematic constraint that can potentially interfere with the estimation of the actual kinematics described in the images. Hyperelastic regularization [Veress et al., 2005; Phatak et al., 2009; Burger et al., 2013] uses a proper strain measure valid for arbitrary large rotations and deformations, but still penalizes strain itself, hence might be considered too strong of a regularization as well.

In this paper, we propose a novel regularizer for finite element-based image registration problems based on the continuum finite strain formulation of the equilibrium gap principle. This regularizer has strong physical basis as it penalizes any deviation from the mechanical equilibrium (which is a fundamental principle) instead of penalizing the kinematics itself (which can be arbitrary). It also benefits from the finite elasticity framework, and does not penalize rigid body displacement or rotation. This work inherits ideas from [Claire et al., 2004; Leclerc et al., 2010], where a similar regularizer has been formulated at the discrete level, and within the linearized elasticity framework. The formulation is presented in details in Section 2, validation is provided in Section 3.1, and an illustration on in vivo cardiac MR images is given in Section 3.2.

\section{Methods}

\subsection{Finite element-based image registration}

\subsubsection{Problem}

Formulation. Let us denote $I_{0} \& I_{t}$ as the scalar intensity fields of two images representing an object occupying the domains $\Omega_{0} \& \Omega_{t}$ in the reference and deformed states, respectively. The problem is to find the smooth mapping $\varphi$, or equivalently the smooth displacement field $\underline{U}(\underline{\varphi}(\underline{X})=\underline{X}+\underline{U}(\underline{X}))$, between $\Omega_{0} \& \Omega_{t}$. This problem is ill-posed, notably because of 
image noise, and must thus be formulated as a regularized minimization problem:

$$
\text { find } \underline{U}=\operatorname{argmin}_{\left\{\underline{U}^{*}\right\}}\left\{J\left(\underline{U}^{*}\right)=(1-\beta) \Psi^{\mathrm{im}}\left(\underline{U}^{*}\right)+\beta \Psi^{\mathrm{reg}}\left(\underline{U}^{*}\right)\right\},
$$

2 where $J$ is the functional to minimize, $\Psi^{\mathrm{im}}$ the image similarity metric or "energy", $\Psi^{\mathrm{reg}}$ the 3 regularization "energy", and $\beta$ is the regularization strength.

4 Similarity metric. We use a simple sum of squares between image intensities, written here 5 in the reference configuration, as similarity metric:

$$
\Psi^{\mathrm{im}}\left(\underline{U}^{*}\right)=\frac{1}{2} \int_{\Omega_{0}}\left(I_{t}\left(\underline{X}+\underline{U}^{*}(\underline{X})\right)-I_{0}(\underline{X})\right)^{2} d \Omega_{0}=\frac{1}{2} \int_{\Omega_{0}}\left(I_{t} \circ \underline{\varphi}^{*}-I_{0}\right)^{2} d \Omega_{0} .
$$

Regularization. Many regularizers have been proposed for image registration problems, including fluid [Christensen et al., 1996] and hyperelastic [Veress et al., 2005; Phatak et al., 2009; Genet et al., 2016] constraints.

In hyperelastic warping [Veress et al., 2005; Phatak et al., 2009], the regularization energy is directly the strain energy of the body:

$$
\Psi^{\text {reg,hyper }}=\int_{\Omega_{0}} \rho_{0} \psi d \Omega_{0},
$$

where $\rho_{0}$ is the mass density, and $\psi$ the specific strain energy potential. Thus, strain, a quantity that we seek to extract from the images and that can be in principle arbitrary, is directly penalized by the regularization in hyperelastic warping. Moreover, the minimizer of Problem (1-3), being the minimum of a "potential" energy, is the solution of a mechanical problem of a body in equilibrium under an unphysical body force that corresponds to the mismatch between the image intensity fields. The obtained deformation therefore has weak mechanical basis.

Motivated by this inconsistency, we propose an alternate regularizer, which essentially penalizes any deviation from the solution of a hyperelastic body in equilibrium with arbitrary boundary loads (but no body load). Let us first recall that mechanical equilibrium, i.e., conservation of momentum, in absence of body load and inertia, can be expressed as:

$$
\left\{\frac{\underline{\operatorname{Div}}}{{ }^{t} \underline{\underline{S}}}=\underline{\underline{\underline{F}}} \cdot \underline{\underline{S}}\right)=0 \quad \forall \underline{X} \in \Omega_{0},
$$

where $\underline{\underline{S}}$ is the second Piola-Kirchhoff stress tensor, and $\underline{\underline{F}}=\frac{\partial \underline{\varphi}}{\partial \underline{X}}$ the transformation gradient [Holzapfel, 2000]. These relations correspond to conservation of linear and angular momentum, respectively. We also recall that the second principle of thermodynamics requires that:

$$
\underline{\underline{S}}=\frac{\partial \rho_{0} \psi}{\partial \underline{\underline{E}}}
$$

where $\underline{\underline{E}}=\frac{1}{2}(\underline{\underline{C}}-\underline{\underline{1}})$ is the Green-Lagrange strain tensor, and $\underline{\underline{C}}={ }^{t} \underline{\underline{F}} \cdot \underline{\underline{F}}$ the right CauchyGreen dilatation tensor [Holzapfel, 2000]. The Green-Lagrange strain tensor is symmetric, 
such that when computed through the constitutive law (5) the second Piola-Kirchhoff stress tensor is necessarily symmetric, and the conservation of angular momentum is automatically verified. However, the conservation of linear momentum still needs to be enforced. In principle one could use $\Psi^{\text {reg,equil }}=\frac{1}{2}\|\underline{\operatorname{Div}}(\underline{\underline{F}} \cdot \underline{\underline{S}})\|_{L^{2}\left(\Omega_{0}\right)}^{2}$. However, we discretize Problem (1) using standard Lagrange elements, so that $\underline{\underline{F}}$ and $\underline{\underline{S}}$ belong to $L^{2}\left(\Omega_{0}\right)$-more strictly they belong to piecewise- $H$ (div; $\left.\Omega_{0}\right)$, but not to $\left.H \overline{\overline{(\operatorname{div}}} ; \Omega_{0}\right)$, as they are $H$ (div) on the elements but have jumps across element faces. Thus, the following equivalent norm is used instead:

$$
\Psi^{\mathrm{reg}, \text { equil }}=\sum_{K} \frac{1}{2}\|\underline{\operatorname{Div}}(\underline{\underline{F}} \cdot \underline{\underline{S}})\|_{L^{2}(K)}^{2}+\sum_{F} \frac{1}{2 h}[[\underline{\underline{F}} \cdot \underline{\underline{S}} \cdot \underline{N}]]_{L^{2}(F)}^{2},
$$

where $K$ denotes the set of elements, $F$ the set the interior faces with normal $\underline{N}$, and $h$ a characteristic length of the finite element mesh. When the mesh is refined, if the discrete stress field converges toward a continuous solution, this discrete norm converges toward the original continuous norm. This regularization is an extension of the equilibrium gap regularization [Claire et al., 2004], but written at the continuous level and valid for arbitrary large deformations.

Mechanical model. As strain energy potential, for both hyperelastic and equilibrated warping we use the classical Ciarlet-Geymonat potential:

$$
\rho_{0} \psi=\frac{\kappa}{2}\left(J^{2}-1-\ln (J)\right)+\frac{\mu}{2}\left(I_{C}-3-2 \ln (J)\right),
$$

where $\kappa \& \mu$ denote bulk and shear modulus, $J=\operatorname{det}(\underline{\underline{F}}), I_{C}=\operatorname{tr}(\underline{\underline{C}}), \underline{\underline{C}}={ }^{t} \underline{\underline{F}} \cdot \underline{\underline{F}}$, $\underline{F}=\underline{1}+\underline{\operatorname{Grad}}(\underline{U})$ [Ciarlet and Geymonat, 1982]. Contrary to the Saint Venant-Kirchhoff potential, the Ciarlet-Geymonat potential is valid for arbitrary deformation levels [Ciarlet and Geymonat, 1982; Le Tallec, 1994]. In practice, we use a unit Young's modulus and null Poisson's ratio, which is equivalent to $\kappa=\frac{1}{3} \& \mu=\frac{1}{2}$, the strength of the regularization being set by the parameter $\beta$.

Let us point out that an incompressible material law could be used to enforce incompressibility. However, that would represent too strong of a kinematic constraint for a problem whose aim is to extract the kinematics from images. Moreover, it is known that the myocardial deformation is not fully incompressible, notably because of perfusion effects [MayNewman et al., 1994]. Nevertheless, if the deformation is indeed incompressible, and that is well represented in the images, the current regularization will not interfere with the image registration, even with a Poisson's ratio of 0 . Indeed, the surface loads will simply adapt themselves so that the deformation of the mesh matches the one in the images.

\subsubsection{Resolution}

Variational formulation. The first variation of Problem (1) yields its variational formulation:

$$
\text { find } \underline{U} \quad / \quad D_{\underline{V}} J(\underline{U})=(1-\beta) D_{\underline{V}} \Psi^{\mathrm{im}}(\underline{U})+\beta D_{\underline{V}} \Psi^{\mathrm{reg}}(\underline{U})=0 \quad \forall \underline{V} \text {, }
$$


1 where

$$
D_{\underline{V}} \Psi^{\mathrm{im}}(\underline{U})=\int_{\Omega_{0}}\left(I_{t} \circ \underline{\varphi}-I_{0}\right)\left(\underline{\nabla I_{t}} \circ \underline{\varphi} \cdot \underline{V}\right) d \Omega_{0}
$$

2 For the case of hyperelastic warping, one obtains:

$$
D_{\underline{\underline{V}}} \Psi^{\text {reg,hyper }}(\underline{U})=\int_{\Omega_{0}} \frac{\partial \rho_{0} \psi}{\partial \underline{\underline{E}}}(\underline{U}): \underline{\underline{\delta E}}(\underline{U}, \underline{V}) d \Omega_{0},
$$

3 where $\underline{\underline{\delta E}}(\underline{U}, \underline{V})=\left({ }^{t} \underline{\underline{F}}(\underline{U}) \cdot \underline{\underline{\operatorname{Grad}}}(\underline{V})\right)_{\text {sym }}$ denotes the variation of Green-Lagrange strain 4 [Holzapfel, 2000]. For the case of equilibrated warping, the following expression results:

$$
\begin{aligned}
D_{\underline{\underline{V}}} \Psi^{\text {reg,equil }}(\underline{U})= & \sum_{K} \int_{K} \underline{\operatorname{Div}}\left(\underline{\underline{F}}(\underline{U}) \cdot \frac{\partial \rho_{0} \psi}{\partial \underline{\underline{E}}}(\underline{U})\right) \\
& \cdot \underline{\operatorname{Div}}\left(\underline{\underline{\operatorname{Grad}}}(\underline{V}) \cdot \frac{\partial \rho_{0} \psi}{\partial \underline{\underline{E}}}(\underline{U})+\underline{\underline{F}}(\underline{U}) \cdot\left(\frac{\partial^{2} \rho_{0} \psi}{\partial \underline{\underline{E}}^{2}}(\underline{U}): \underline{\underline{E}}(\underline{U}, \underline{V})\right)\right) d K \\
+ & \sum_{F} \int_{F}\left[\left[\left(\left[\underline{\underline{F}}(\underline{U}) \cdot \frac{\partial \rho_{0} \psi}{\partial \underline{\underline{E}}}(\underline{U})\right) \cdot \underline{N}\right]\right]\right. \\
\cdot & {\left[\left[\left(\underline{\underline{\operatorname{Grad}}}(\underline{V}) \cdot \frac{\partial \rho_{0} \psi}{\partial \underline{\underline{E}}}(\underline{U})+\underline{\underline{F}}(\underline{U}) \cdot\left(\frac{\partial^{2} \rho_{0} \psi}{\partial \underline{\underline{E}}^{2}}(\underline{U}): \underline{\underline{\delta E}}(\underline{U}, \underline{V})\right)\right) \cdot \underline{N}\right]\right] d F . }
\end{aligned}
$$

5

6 Linearization. Nonlinear Problem (8) is solved iteratively using Newton's method, so that 7 at each iteration the following linearized problem must be solved:

$$
\text { find } \begin{aligned}
\underline{\Delta U} / \quad(1-\beta) a^{\mathrm{im}}(\underline{U} ; \underline{\Delta U}, \underline{V})+\beta a^{\mathrm{reg}}(\underline{U} ; \underline{\Delta U}, \underline{V}) \\
=(1-\beta) b^{\mathrm{m}}(\underline{U} ; \underline{V})+\beta b^{\mathrm{reg}}(\underline{U} ; \underline{V}) \forall \underline{V},
\end{aligned}
$$

8 where (without image Hessian terms)

$$
\left\{\begin{array}{l}
a^{\mathrm{im}}(\underline{U} ; \underline{\Delta U}, \underline{V})=\int_{\Omega_{0}}\left(\left(\underline{\nabla I_{t}} \circ \underline{\varphi}(\underline{U})\right) \cdot \underline{\Delta U}\right)\left(\left(\underline{\nabla I_{t}} \circ \underline{\varphi}(\underline{U})\right) \cdot \underline{V}\right) d \Omega_{0} \\
b^{\mathrm{im}}(\underline{U} ; \underline{V})=\int_{\Omega_{0}}\left(\left(I_{t} \circ \underline{\varphi}(\underline{U})\right)-I_{0}\right)\left(\left(\underline{\nabla I_{t}} \circ \underline{\varphi}(\underline{U})\right) \cdot \underline{V}\right) d \Omega_{0}
\end{array} .\right.
$$

9 For the case of hyperelastic warping, one has:

$$
\left\{\begin{array}{c}
a^{\text {reg,hyper }}(\underline{U} ; \underline{\Delta U}, \underline{V})=\int_{\Omega_{0}} \frac{\partial \rho_{0} \psi}{\partial \underline{\underline{E}}}(\underline{U}): \underline{\underline{\delta E}}(\underline{\Delta U}, \underline{V}) \\
+\underline{\underline{\delta E}}(\underline{U}, \underline{\Delta U}): \frac{\partial^{2} \rho_{0} \psi}{\partial \underline{\underline{E}}^{2}}(\underline{U}): \underline{\underline{E E}}(\underline{U}, \underline{V}) d \Omega_{0} \\
b^{\text {reg,hyper }}(\underline{U} ; \underline{V})=\int_{\Omega_{0}} \frac{\partial \rho_{0} \psi}{\partial \underline{\underline{E}}}(\underline{U}): \underline{\underline{\delta E}}(\underline{U}, \underline{V}) d \Omega_{0}
\end{array}\right.
$$


1 And for the case of equilibrated warping, one obtains:

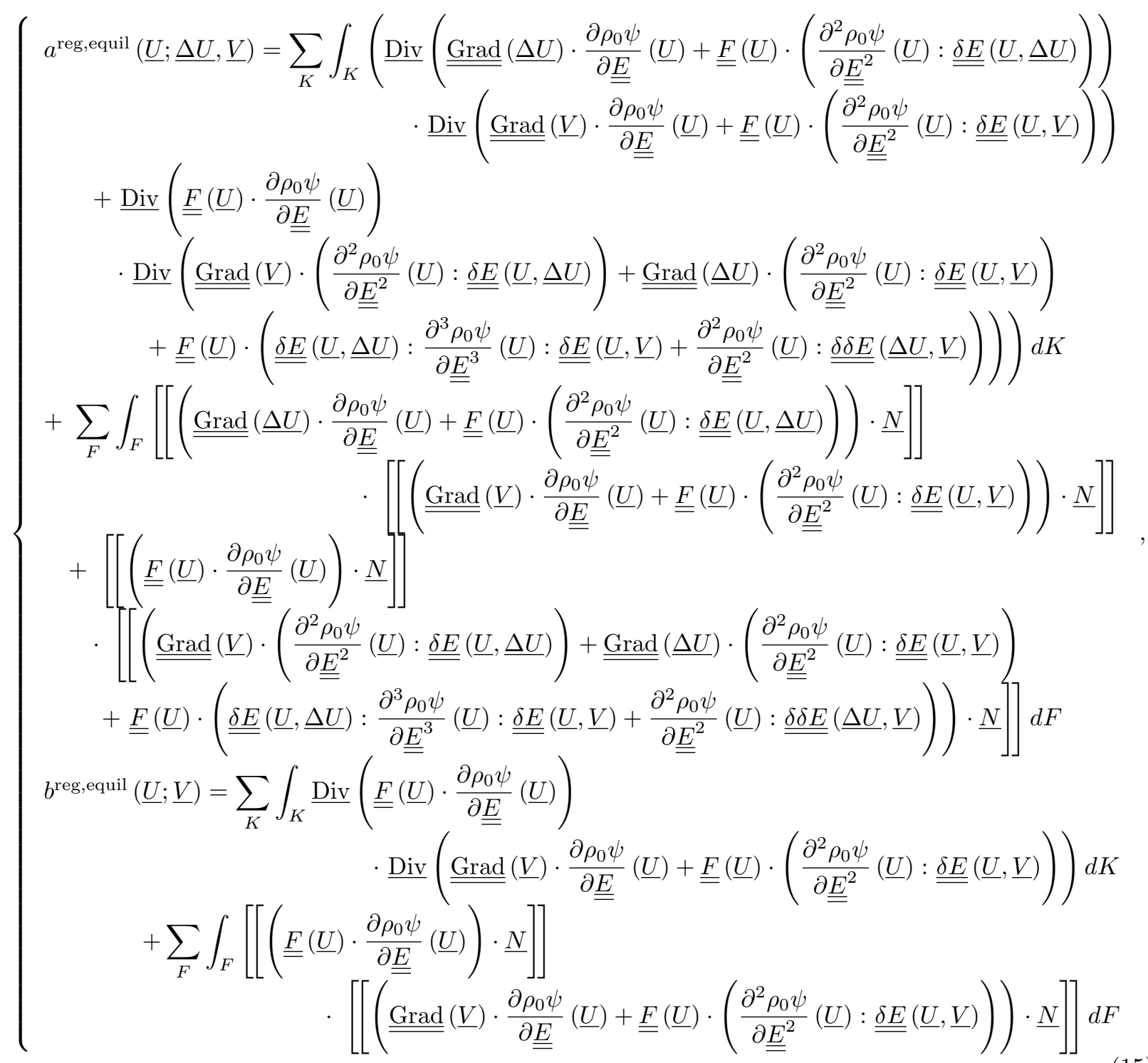

2 where $\underline{\underline{\delta \delta E}}(\underline{\Delta U}, \underline{V})=\left({ }^{t} \underline{\underline{\operatorname{Grad}}}(\underline{\Delta U}) \cdot \underline{\underline{\operatorname{Grad}}}(\underline{V})\right)_{\text {sym }}$ denotes the second variation of Green3 Lagrange strain [Holzapfel, 2000].

4 Discretization. Problem (12) is discretized using standard continuous Galerkin elements, 5 yielding the discrete problem:

$$
\text { find } \underline{\Delta \mathbb{U}} \quad / \quad\left((1-\beta) \underline{\underline{\mathbb{A}^{i m}}}(\underline{\mathbb{U}})+\beta \underline{\underline{\mathbb{A}^{\text {reg }}}}(\underline{\mathbb{U}})\right) \cdot \underline{\Delta \mathbb{U}}=(1-\beta) \underline{\underline{\mathbb{B}^{i m}}}(\underline{\mathbb{U}})+\beta \underline{\underline{\mathbb{B}^{\text {reg }}}}(\underline{\mathbb{U}})
$$

6 Nonlinear solver. For a given couple of images, the discrete Problem (16) is iterated un7 til convergence. In practice, convergence is achieved when the incremental error (i.e., $8\|\underline{U}\| /\|\underline{U}\|)$ reaches $1 \%$. For time series of images, each image $t$ is registered to the ref9 erence image 0 , taking the converged solution of the previous image $t-1$ as initialization of the nonlinear iterations for the current image $t$. 
Gradient-free line search. The solution of Problem (8) has proven unstable, especially on in vivo images, notably (i) because of the steepness of the mechanical term, and (ii) because in the current VTK implementation, interpolated gradients are not the actual gradients of the interpolated images ${ }^{1}$ (so that the minimum of the image similarity energy does not correspond to the root of its "derivative"). Thus, a gradient-free golden section line search [Press et al., 2007] has been implemented, where the initial interval is taken as $\left[\frac{1-\varphi}{2-\varphi} ; \frac{1}{2-\varphi}\right]$ (with $\varphi=\frac{1+\sqrt{5}}{2}$ ) such that the two initially tested values are $0 \& 1$. This line search comes after the search direction as been computed by solving Problem (16), and allows to find the optimal relaxation parameter at each iteration.

Image scaling. Since the image norm used here is sensitive to global image intensity that changes from scan to scan and, for tagged images, that decreases slowly throughout the cardiac cycle, we introduce a global image intensity scaling. Because the decrease is slow, we use an explicit scaling. Specifically, after registering the image $t$ to the reference image 0 , we compute the linear scaling that best matches both images:

$$
\text { find }(a, b)=\operatorname{argmin}_{\mathbb{R}^{2}}\left\{J^{2}\left(a^{*}, b^{*}\right)=\frac{1}{2} \int_{\Omega_{0}}\left(\left(a^{*}\left(I_{t} \circ \underline{\varphi}\right)+b^{*}\right)-I_{0}\right)^{2} d \Omega_{0}\right\},
$$

which leads to the following symmetric linear system:

$$
\left(\begin{array}{cc}
\int_{\Omega_{0}}\left(I_{t} \circ \underline{\varphi}\right)^{2} d \Omega_{0} & \int_{\Omega_{0}}\left(I_{t} \circ \underline{\varphi}\right) d \Omega_{0} \\
\int_{\Omega_{0}}\left(I_{t} \circ \underline{\varphi}\right) d \Omega_{0} & 1
\end{array}\right) \cdot\left(\begin{array}{c}
a \\
b
\end{array}\right)=\left(\begin{array}{c}
\int_{\Omega_{0}} I_{0} \cdot\left(I_{t} \circ \underline{\varphi}\right) d \Omega_{0} \\
\int_{\Omega_{0}} I_{0} d \Omega_{0}
\end{array}\right)
$$

This scaling is then used for the registration of the subsequent image $t+1$ to the reference image 0 .

\subsubsection{Implementation}

The time stepping, nonlinear solver and gradient-free line search have been implemented in python ${ }^{2}$. The finite element procedure is directly based on the FEniCS library ${ }^{3}$, which provides automatic derivation and integration of the mechanical terms (14) or (15): as FEniCS can process symbolic expressions, it can determine the degree of the expressions it is given, and thus determine the order of the quadrature required to exactly integrate these expressions [Logg et al., 2012; Alnæs et al., 2015]. Integration of image terms (13) is done with the help of the VTK library ${ }^{4}$ for image derivation and interpolation [Schroeder et al., 2006], using linear interpolation of image intensity and image gradients. High-order quadrature is used for integration of image terms, in order to have as many integration

\footnotetext{
${ }^{1}$ http://www.vtk.org/Wiki/VTK/Image_Interpolators\#Gradients

${ }^{2}$ https://www.python.org

${ }^{3}$ https://www.fenicsproject.org

${ }^{4}$ http: //www.vtk.org
} 
1 points as pixels in the mesh. For greater efficiency, the $\mathrm{C}++$ interface to both FEniCS \& 2 VTK is used for the integration of image terms. The code is freely available at https: 3 //gitlab.inria.fr/mgenet/dolfin_dic.

4 2.2. Image synthesis, acquisition, combination and segmentation

5 2.2.1. Image synthesis

$6 \quad$ In order to validate our method, we generated a series of 2D synthetic tagged MR images.

7 The reference image has the following intensity field:

$$
I_{0}(\underline{X})=\sqrt{\left|\sin \left(\frac{\pi X_{0}}{s}\right)\right| \cdot\left|\sin \left(\frac{\pi X_{1}}{s}\right)\right|},
$$

8 where $X_{0} \& X_{1}$ denote spatial coordinates, and $s$ is the tagging period. The prescribed 9 deformation is a simple uniaxial and uniform deformation:

$$
\left\{\begin{array}{l}
x_{0}=\sqrt{2 f(t)+1} X_{0} \\
x_{1}=X_{1}
\end{array},\right.
$$

where $f(t)$ is a temporal function. Correspondingly,

$$
\underline{\underline{F}}=\frac{\partial \underline{x}}{\partial \underline{X}}=\left(\begin{array}{cc}
\sqrt{2 f(t)+1} & 0 \\
0 & 1
\end{array}\right),
$$

11

$$
\underline{\underline{C}}={ }^{t} \underline{\underline{F}} \cdot \underline{\underline{F}}=\left(\begin{array}{cc}
2 f(t)+1 & 0 \\
0 & 1
\end{array}\right)
$$

12 and

$$
\underline{\underline{E}}=\frac{\underline{\underline{C}}-\underline{\underline{\underline{1}}}}{2}=\left(\begin{array}{cc}
f(t) & 0 \\
0 & 0
\end{array}\right) .
$$

Because the mapping is uniform, one simply has $\underline{x}=\underline{\underline{F}} \cdot \underline{X}$. The deformed images therefore have the following intensity:

$$
I_{t}(\underline{x}, t)=I_{0}\left(\underline{F}^{-1} \cdot \underline{x}\right) .
$$

In practice, we generated images of size $1 \times 1$ (arbitrary units) with $100 \times 100$ pixels, $s=0.1$ (arbitrary units), 21 frames over $[0 ; 1]$, and $f(t)=-0.30 t$. Normal noise with zero mean and standard deviation of 0.1 (i.e., signal-to-noise ratio of 10) and 0.2 (i.e., signal-to-noise ratio of 5) was also added.

To process the synthetic images, simple square meshes with multiple element sizes were generated using GMSH ${ }^{5}$ [Geuzaine and Remacle, 2009].

\footnotetext{
${ }^{5}$ http://gmsh.info
} 


\subsubsection{Image acquisition and combination}

Image acquisition. Standard multi-slice balanced Steady State Free Precession (SSFP) cine and whole-heart 3D Complimentary SPAtial Modulation of Magnetization (3D CSPAMM) [Rutz et al., 2008; Stoeck et al., 2012] images were acquired in a healthy volunteer (female, age 27) on a clinical 1.5T MR scanner (Philips Achieva, Best, The Netherlands). Multi-slice balanced SSFP cine images were acquired with a spatial resolution of $1.2 \times 1.2 \times 8 \mathrm{~mm}^{3}$ and a temporal resolution of $35 \mathrm{~ms}$. 3D CSPAMM images were acquired with a spatial resolution of $3.5 \times 7.7 \times 7.7 \mathrm{~mm}^{3}$ reconstructed to $1 \times 1 \times 1 \mathrm{~mm}^{3}$ through zero-filling in Fourier space (which corresponds to a cardinal sine interpolation in image space), a tag wave modulation period of $14 \mathrm{~mm}$ (i.e., tagging distance of $7 \mathrm{~mm}$ in the magnitude images) and a temporal resolution of $28 \mathrm{~ms}$. Image acquisition was performed during respiratory navigator gated breath-holding, and geometrical stack alignment of all tagged volume images was performed by incorporating navigator offsets and rigid image registration. More details on the imaging process can be found in [Rutz et al., 2008; Stoeck et al., 2012].

Image combination. Cine images were normalized using their maximal pixel intensity. Moreover, they were resampled in order to have an isotropic pixel size, ensuring a correct integration of image terms (as detailed Section 2.1.3, the quadrature of image terms is chosen such that in average there are as many integration points as pixels in each element-better quadrature should be used to correctly handle images with anisotropic pixel sizes [Pierré et al., 2016]). This processing was done using MeVisLab ${ }^{6}$.

3D CSPAMM magnetic resonance imaging produces six time series of volumetric images: magnitude and "phase" for three orthogonal tag orientations [Rutz et al., 2008; Stoeck et al., 2012]. Initial magnitude images are modulated by $|\sin (\underline{k} \underline{x})|$, where $\underline{k}$ is the wave vector of the modulation. Thus they are not continuously differentiable, which could be an issue since the registration method involves image gradients. On the other hand, "phase" images simply contain the sign of the modulation, i.e., $\operatorname{sign}(\sin (\underline{k} \underline{x}))$. While in principle it would be possible to reconstruct time series of continuously differentiable images $(\operatorname{sign}(\sin (\underline{k} \underline{x})) \cdot|\sin (\underline{k} \underline{x})|=\sin (\underline{k} \underline{x})$ is indeed differentiable), in practice, however, such smooth reconstruction is not possible because of noise. Nevertheless, magnitude images are smooth enough to be used directly for the registration. Thus, the three time series of orthogonal tagged images were combined into a single time series:

$$
I=\sqrt[3]{I_{1} I_{2} I_{3}}
$$

where the cube root introduces another source of theoretical non differentiability that, in practice, does not generate issues as the non differentiability is smoothed out by the image sampling. More importantly, this allows to keep a reasonable contrast in the combined images. Similar to untagged images, the combined tagged images were normalized using their maximal pixel intensity.

\footnotetext{
${ }^{6}$ http: //www.mevislab.de
} 


\subsubsection{Image segmentation}

The left ventricle was manually segmented on the cine and 3D CSPAMM images using MeVisLab. For cine images, the first frame was used, i.e., end-diastole. For tagged images, the last frame (which is not exactly end-diastole since the last part of the cardiac cycle is not imaged [Rutz et al., 2008; Stoeck et al., 2012]) was used because of absent blood pool signal and hence better contrast between myocardium and lumen. The surface meshes generated by MeVisLab were then used to generate volume meshes using GMSH, which first reparametrizes the surface meshes, remeshes them with proper finite elements, and then generates the volume meshes [Geuzaine and Remacle, 2009; Remacle et al., 2010]. For the sake of simplicity, first order finite elements were used, though the method can be used with higher order elements in order to improve convergence. Finally, a local pseudo-prolate spheroidal coordinate system was assigned to all nodes and elements of the mesh to define local transmural, circumferential and longitudinal directions. More details on the pipeline for reconstruction of the left ventricular mesh can be found in [Genet et al., 2014, 2015].

For finite element-based image registration on untagged images, it is necessary for the mesh to extend on both sides of the object boundary if one wants to track its motion in both directions. Indeed, the correlation only happens under the mesh, so that if the boundary moves toward the outward normal (i.e., away from the mesh), and if there is no contrast in the image under the mesh, then the boundary motion will not be tracked. (If the boundary moves toward the inward normal, then it will be tracked.) Thus, for cine images, a layer of elements was added around the mesh using GMSH, which has the ability to extrude surfaces in the direction of their normal [Geuzaine and Remacle, 2009]. These elements are used to enhance the image registration but not for strain analysis.

For image registration on tagged images, the registration is run backwards in time, i.e., from the last to the first image in the series, since the mesh is actually created on the last image. As a consequence, strains are naturally defined from the last image, which is usually around mid-diastole [Rutz et al., 2008; Stoeck et al., 2012]. In order to express strains with respect to end-diastole, i.e., the first image of the series, we need to multiplicatively combine the transformation gradients:

$$
\underline{\underline{F_{0 \rightarrow t}}}=\underline{\underline{F_{T \rightarrow t}}} \cdot \underline{\underline{F_{0 \rightarrow T}}}=\underline{\underline{F_{T \rightarrow t}}} \cdot \underline{\underline{F_{T \rightarrow 0}}}{ }^{-1},
$$

where $\underline{F}_{0 \rightarrow t}$ is the gradient of the mapping from the first image 0 to the current image $t, F_{T \rightarrow t}$ is the gradient of the mapping from the last image $T$ to the current image $t$, and $\underline{F_{0 \rightarrow T}} \overline{\text { is the }}$ gradient of the mapping from the first to the last image, i.e., of the inverse mapping from the last to the first image. Note that both $F_{T \rightarrow t}$ and $F_{T \rightarrow 0}$ are computed during the registration process, so that $F_{0 \rightarrow t}$ can be computed explicitly from Equation (26). Then, Green-Lagrange strains with end-diastole as reference configuration can be directly computed from $\underline{\underline{F_{0 \rightarrow t}}}$. 


\section{Results \& Discussion}

\subsection{Validation on in silico images}

\subsubsection{Influence of regularization strength}

We first consider the simple problem of a uniformly deforming image (see Section 2.2 for details on images synthesis), and study the influence of regularization type (hyperelastic vs. equilibrated), as well as regularization strength $\beta$, on the computed strains. Since the images are $2 \mathrm{D}$, a plane strain assumption is made in the mechanical model used in the regularization. Figure 1 shows the deforming images (for different noise and deformation levels) with superimposed warped meshes (for both hyperelastic and equilibrated warping). Regularization strength was $\beta=0.1$ for all cases. One can see that equilibrated warping provides almost perfect registration, while hyperelastic warping results in underestimated and more noisy strains.

This is better seen in Figure 2, which shows the final computed longitudinal strain (mean \pm standard deviation over the mesh) for both methods and for the different noise levels. We consider Green-Lagrange strain, the reference configuration being the undeformed mesh, which corresponds to the initial undeformed image. Ground truth is $-30 \%$ homogeneous longitudinal strain, all other components being null. In the case of hyperelastic warping, the mesh does not deform at all if the regularization strength $\beta$ is close to 1 , because the hyperelastic regularization basically makes the mesh extremely stiff. When the regularization strength $\beta$ decreases, the computed strain tends to converge toward the exact value. For noise-free images, it does converge exactly toward ground truth. For noisy images, there is an optimum (here $c a . \beta=0.1$ ) for which the mean strain is closest to the exact value and standard deviation is still limited; however, further reduction in the regularization strength leads to divergence of the mean and the standard deviation from ground truth. Conversely, in case of equilibrated warping, the registration is almost perfect, over a wide range of regularization strengths. For noise-free images, it is actually perfect for all regularization strengths. For noisy images, as long as there is enough regularization (here $c a . \beta>0.001$ ) to get the registration to converge, the registration is almost perfect.

Similar conclusions can be drawn from Figure 3, which shows the normalized image similarity root mean square error (RMSE) at the last time frame $T$, i.e.

$$
\operatorname{RMSE}=\frac{\sqrt{\Psi^{\mathrm{im}}}}{\sqrt{\int_{\Omega_{0}} I_{0}^{2} d \Omega_{0}}}=\frac{\sqrt{\int_{\Omega_{0}}\left(I_{T} \circ \underline{\varphi}-I_{0}\right)^{2} d \Omega_{0}}}{\sqrt{\int_{\Omega_{0}} I_{0}^{2} d \Omega_{0}}},
$$

as a function of regularization strength $\beta$. For large values of regularization strength, hyperelastic warping heavily degrades the registration, and the image similarity error is largely above the noise level. When decreasing the regularization strength, the image similarity error converges toward noise level. (The convergence is slower for noise-free images, simply because for noisy images the error is noise-dominated for $\beta<0.1$.) Conversely, with 



$$
E_{00}=0 \%
$$

$$
E_{00}=-15 \%
$$

$$
E_{00}=-30 \%
$$

Figure 1: Results on synthetic data. Uniformly deformed images (exact strain is thus homogeneous) with superimposed meshes obtained with equilibrated and hyperelastic warping. Each row shows a different noise level $(0.0,0.1 \& 0.2)$, while each column shows a different deformation level $(0 \%,-15 \% \&-30 \%)$. Results for equilibrated warping are shown in red, blue \& green, while results for hyperelastic warping are shown in cyan, orange \& magenta. Regularization strength was $\beta=0.1$ for all cases. One can see that equilibrated warping provides almost perfect registration, while hyperelastic warping provides underestimated and more noisy strains. 

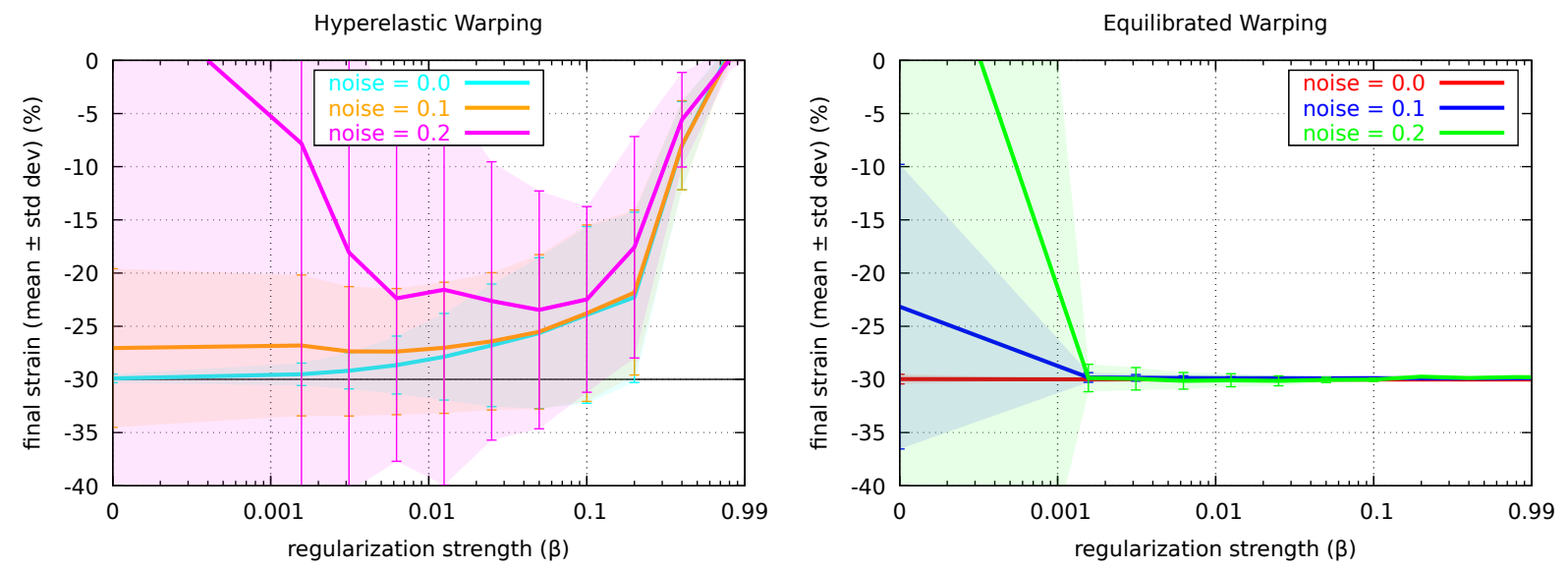

Figure 2: Results on synthetic data. Final measured longitudinal Green-Lagrange strain (mean \pm standard deviation over the mesh) as a function of regularization strength $\beta$, for hyperelastic (left) and equilibrated (right) warping, and for different noise levels $(0.0,0.1 \& 0.2)$. Ground truth is $-30 \%$ homogeneous strain. Hyperelastic regularization has a very strong impact on registration, and is very sensitive to regularization strength. Conversely, equilibrium gap regularization has a much reduced impact on registration.

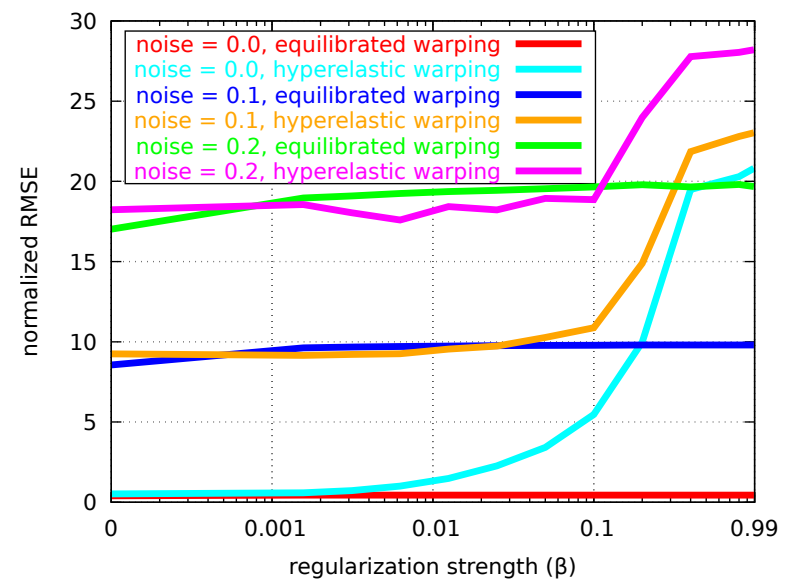

Figure 3: Results on synthetic data. Final normalized image similarity error as a function of regularization strength $\beta$, for equilibrated and hyperelastic warping, and for different noise levels. Again, hyperelastic regularization has a very strong impact on registration, contrary to equilibrium gap regularization. 
equilibrated warping, the image similarity error is close to noise level independently of the regularization strength. It is interesting to see that for small values of regularization strength, the image similarity error is close to noise level even though the actual strain is far from ground truth, meaning that image similarity error is not a good criterion for the success of the registration.

This result illustrates a general weakness of the hyperelastic regularization, and the main strength of the equilibrium gap regularization. Indeed, the exact uniform strain is not a minimizer of the hyperelastic energy, but it is a minimizer of the equilibrium gap energy. Actually the unique minimizer of the hyperelastic energy is the null deformation, so that strain measurements are always compromised. Conversely, any deformation that corresponds to the solution of a hyperelastic body in equilibrium with surface loads is a minimizer of the equilibrium gap energy, so that it minimally affects strain measurements.

\subsubsection{Influence of mesh density}

We now study the influence of mesh density on the registration. The mesh used in the previous section was successively refined until the elements reached the image pixel size. They are shown in the left column of Figure 4, while the middle and right column show the registration results (final strain as a function of regularization strength, similar to Figure 2) for hyperelastic and equilibrated warping, respectively.

When the mesh is refined, the signal-to-noise ratio on each finite element decreases, and larger regularization strengths are required to perform the registration and obtain satisfying strains. As a result, the range of registration strengths that provide satisfying strains becomes smaller. This is the case for both regularization methods; however, this is more critical for hyperelastic regularization, for which the range of acceptable regularization strengths becomes very narrow. Conversely, for equilibrium gap regularization, the impact of mesh size is less critical.

Further validation of the proposed method based on the Cardiac Motion Tracking Challenge [Tobon-Gomez et al., 2013] is provided in Appendix A.

\subsection{Results on in vivo images}

We now consider in vivo tagged and untagged cardiac MR images of a healthy human subject (see Section 2.2 for details on image acquisition, combination and segmentation).

\subsubsection{Results on in vivo tagged images}

Finding the optimal regularization strength. Similarly to Figure 2 for synthetic images, Figure 5 shows the influence of regularization strength $\beta$ on strain extracted from tagged images using equilibrated warping. Here we consider Green-Lagrange strains at end-systole with regard to end-diastole, rotated in local pseudo-prolate spheroidal coordinates (transmural, circumferential, longitudinal), and we show the mean \pm standard deviation across the entire ventricle. For circumferential and longitudinal components, as well as for the circumferential-longitudinal shear component (i.e., the out-of-plane twist), the trend is similar to that found with the in silico images: above a given regularization strength (here $c a$. 
Mesh
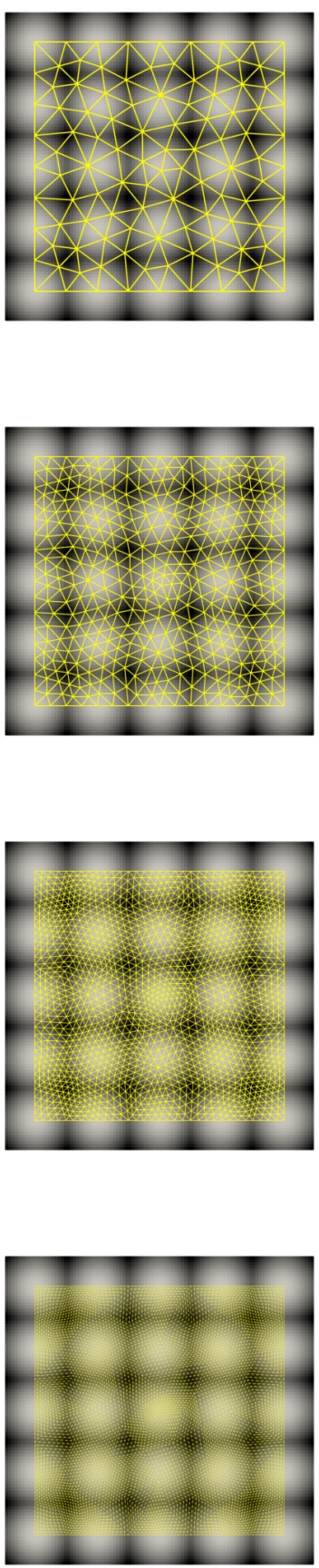

Hyperelastic warping
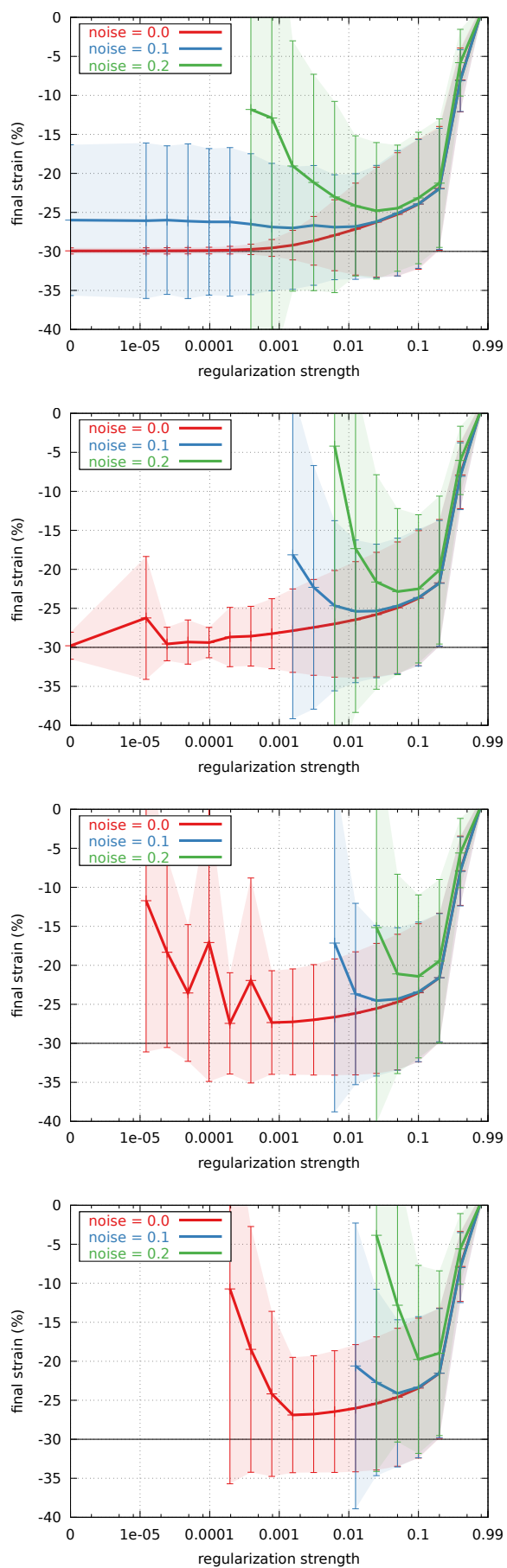

Equilibrated warping
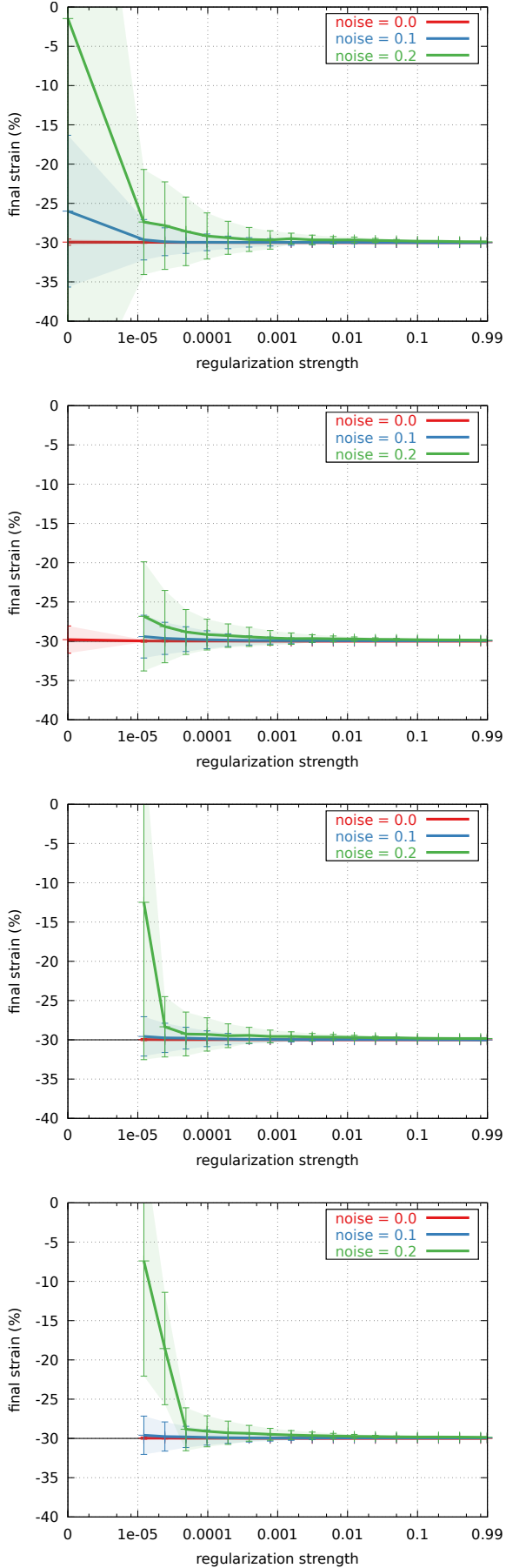

Figure 4: Results on synthetic data. Each line corresponds to a mesh refinement level. Left column shows the mesh, while middle and right columns show registration results (final strain as a function of regularization strength, similar to Figure 2) for hyperelastic and equilibrated warping, respectively. Failed computations (because of divergence or large unrealistic strains) have been removed. For both regularization methods, stronger regularization is required for finer meshes. However, the impact of mesh size is more benign for equilibrated warping compared to hyperelastic warping. 
$\beta=0.1$ ) the equilibrium gap regularization does not significantly affect the mean computed strains, though increasing the regularization strength does reduce their standard deviation. Conversely, radial strain is not very well captured and is largely underestimated - decreasing regularization strength does increase mean radial strain toward more realistic values, but at the cost of a very large standard deviation.

Strain measurement. Figure 6 shows the results of the registration (for $\beta=0.1$ ), with 3D CSPAMM images superimposed with the deformed mesh. The motion of the left ventricle is very well tracked. Figure 7 shows the temporal variations of each strain component averaged over the entire ventricle. Figure 8 shows the spatial variations of each strain component at end-systole, averaged over multiple sectors. Once again, we see that circumferential \& longitudinal shortenings are very well captured, as well as circumferential-longitudinal (i.e., out-of-plane) twist. Moreover, these components are mostly homogeneous within the ventricle, as expected for a healthy heart [Moore et al., 2000]. Furthermore, longitudinal variation of radial-circumferential (i.e., in-plane) twist, with positive rotation differential between endocardium and epicardium at apex and negative rotation differential at base [Bertini et al., 2010], was correclty captured. Conversely, radial thickening is largely underestimated. This has already been noted in [Tobon-Gomez et al., 2013], using other registration methods. It is due to the limited spatial resolution of $3.5 \times 7.7 \times 7.7 \mathrm{~mm}^{3}$, which leads to partial voluming at the endo- and epicardial surfaces and thus reduced accuracy of in-plane motion tracking. We are currently working on characterizing the impact of image resolution on measured radial strains.

\subsubsection{Results on in vivo untagged images}

Finding the optimal regularization strength. We now show similar results for untagged images. Figure 9 is analogous to Figure 5, and shows end-systolic strain as a function of regularization strength. Impact of equilibrium gap regularization on average circumferential \& longitudinal strains is again limited, albeit slightly larger than for tagged images, and without clear convergence of the mean value in the radial strain. Moreover, except for very large regularization strength, there is a spurious radial-longitudinal deformation, which is located toward the free wall of the ventricle. This is due to the fact that, besides lack of texture, short-axis cine stacks have very poor longitudinal resolution, so that tracking of the myocardium, especially toward the base, is not robust. We are currently working on combining both short-axis and long-axis cine images in order to overcome this difficulty.

Strain measurement. Figures 10, $11 \& 12$ show, for $\beta=0.1$, cine images superimposed with the deformed mesh, temporal and spatial variations of strains, respectively. As opposed to that found in the tagged images, radial thickening is very well captured by the equilibrated warping method. The method was even able to capture regional variations of radial strain: it is larger in the left ventricular free wall than in the septum [Moore et al., 2000], as a consequence of being pressurized on both side. Circumferential and longitudinal shortenings are also well captured. Despite the spurious radial-longitudinal strain, it is interesting that the method was able to capture some in-plane (i.e., radial-circumferential) and out-ofplane (i.e., circumferential-longitudinal) twists, even though there is no contrast within the 

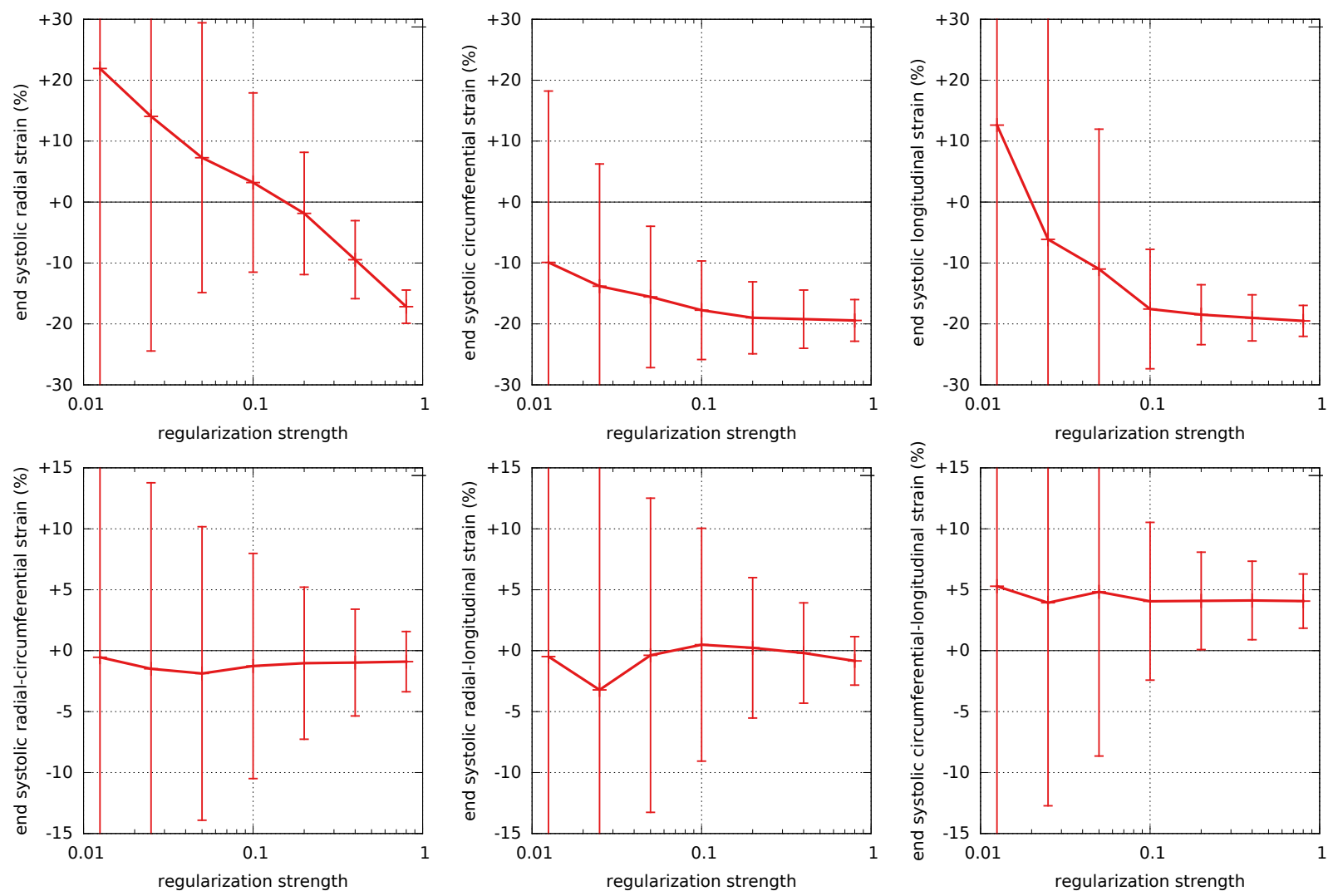

Figure 5: Results on in vivo tagged data. Green-Lagrange strain components at end-systole with respect to end-diastole (mean \pm standard deviation over the ventricle) as a function of regularization strength. Except for radial strain (top left), for all other components, including circumferential (top center), longitudinal (top right) and circumferential-longitudinal (bottom right) components, the equilibrium gap regularization has a limited impact on the registration above a certain level of regularization required to regularize the registration problem. 

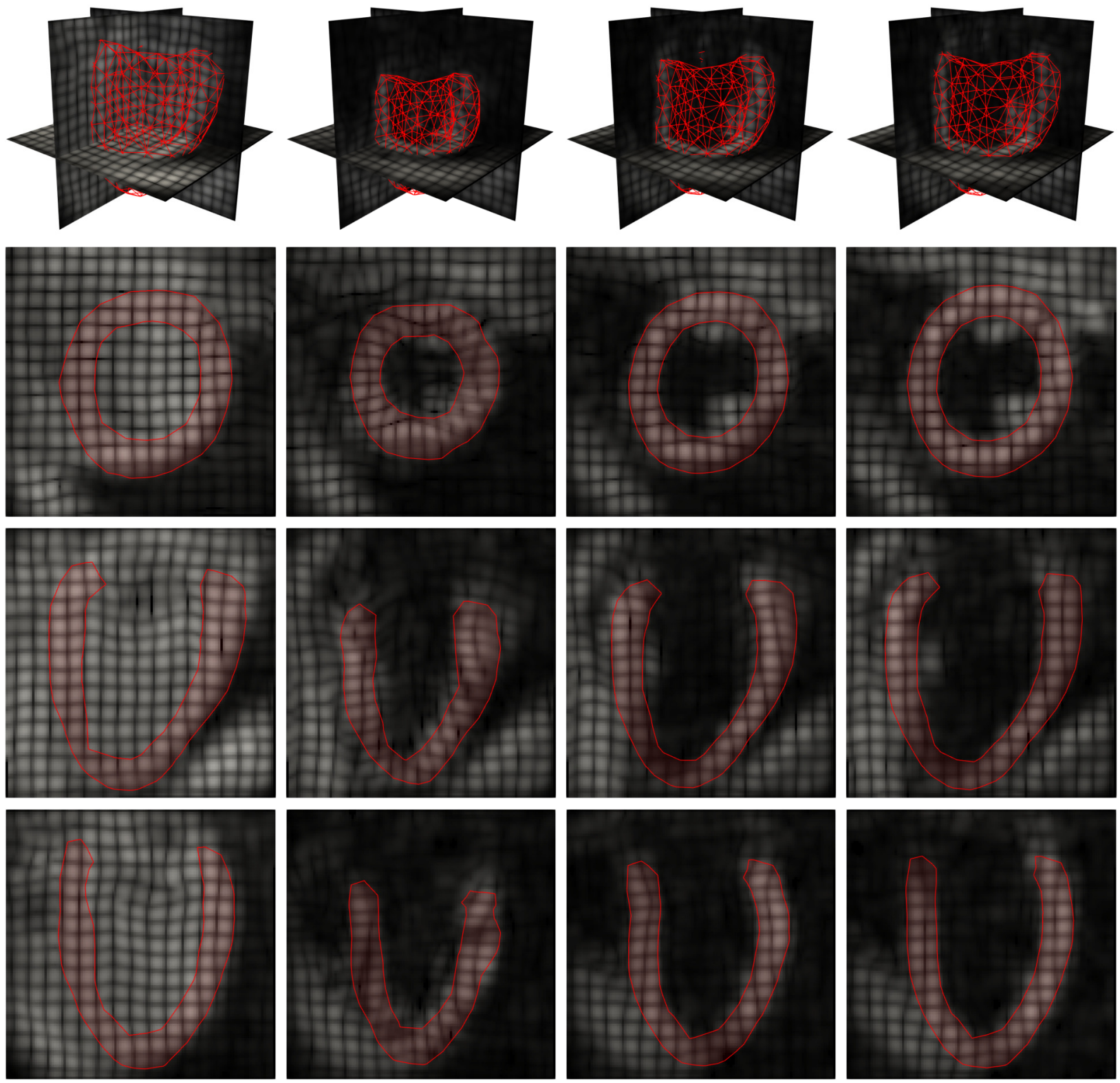

$t=17 m s$

$$
t=251 \mathrm{~ms}
$$

$t=486 \mathrm{~ms}$

$t=720 \mathrm{~ms}$

Figure 6: Results on in vivo tagged data. Sequence of 3D CSPAMM images with superimposed warped mesh, for a regularization strength of $\beta=0.1$. First row shows $3 \mathrm{D}$ view, while second, third and fourth rows show short axis, two-chamber and four-chamber views, respectively. 

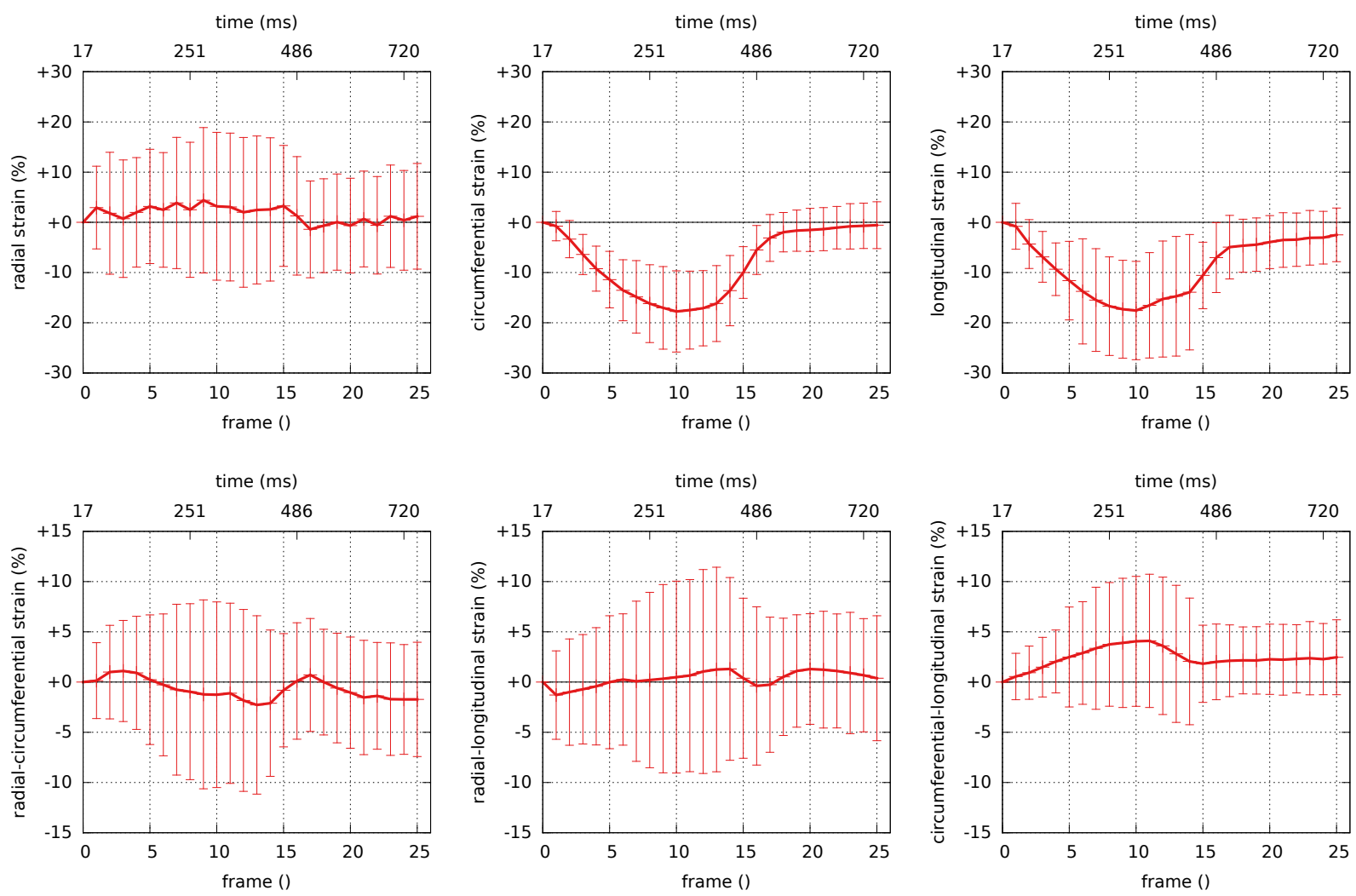

Figure 7: Results on in vivo tagged data. Green-Lagrange strain components with respect to end-diastole (mean \pm standard deviation over the ventricle) as a function of time, for a regularization strength of $\beta=0.1$. Circumferential \& longitudinal shortenings, as well as circumferential-longitudinal (i.e., out-of-plane) twist are well captured. Conversely, radial thickening is largely underestimated. 

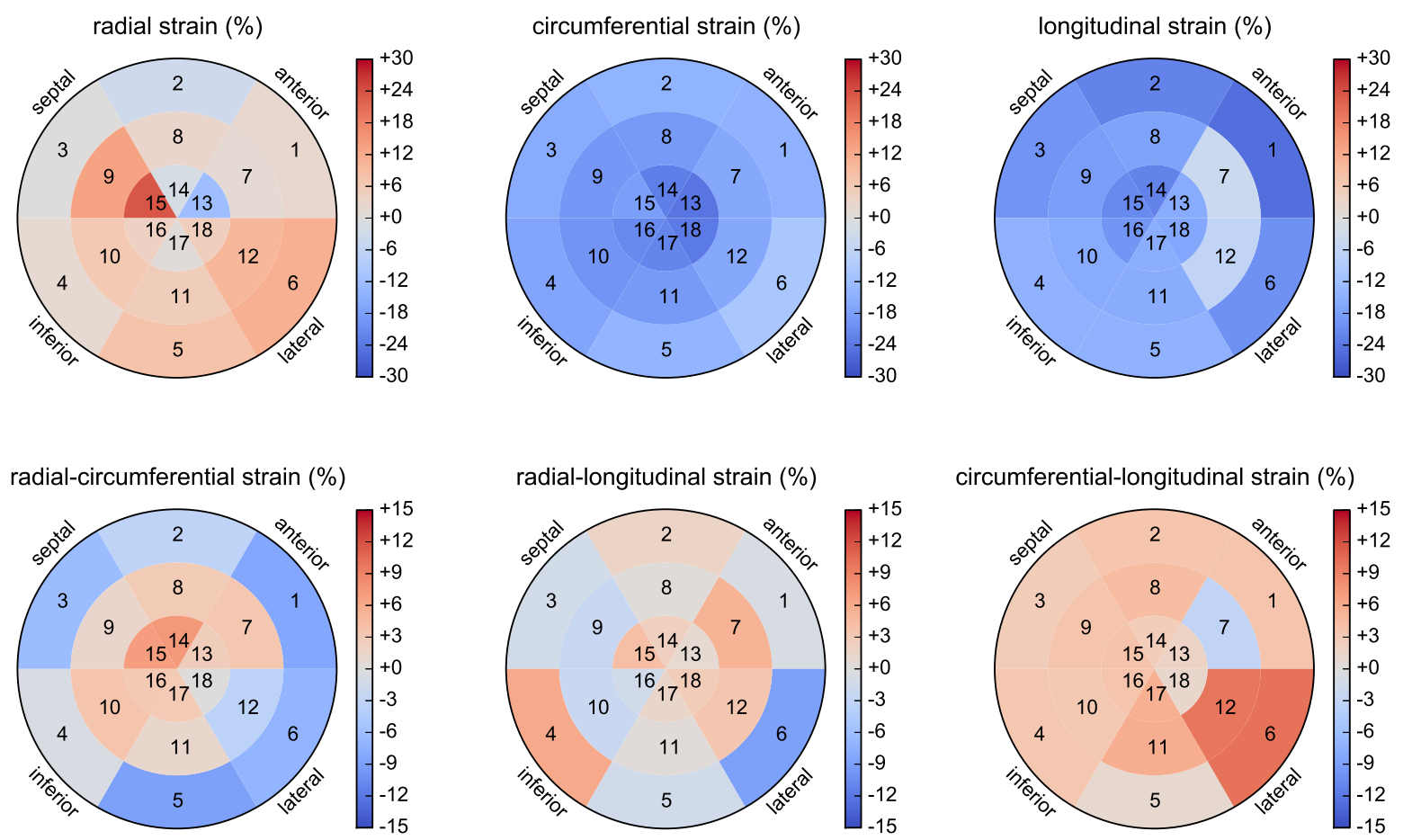

Figure 8: Results on in vivo tagged data. Bull's eye plots of end-systolic Green-Lagrange strains, for a regularization strength of $\beta=0.1$. The left ventricle was split into 18 sectors $(6$ circumferential and 3 longitudinal sections, see Figure 12), and strains were averaged over each sector. Numbers within sectors represent their ID, as described on Figure 12. Circumferential, longitudinal and circumferential-longitudinal components are mostly homogeneous within the ventricle, which is expected for a normal case [Moore et al., 2000]. Longitudinal variation of radial-circumferential shear is captured as well [Bertini et al., 2010]. 

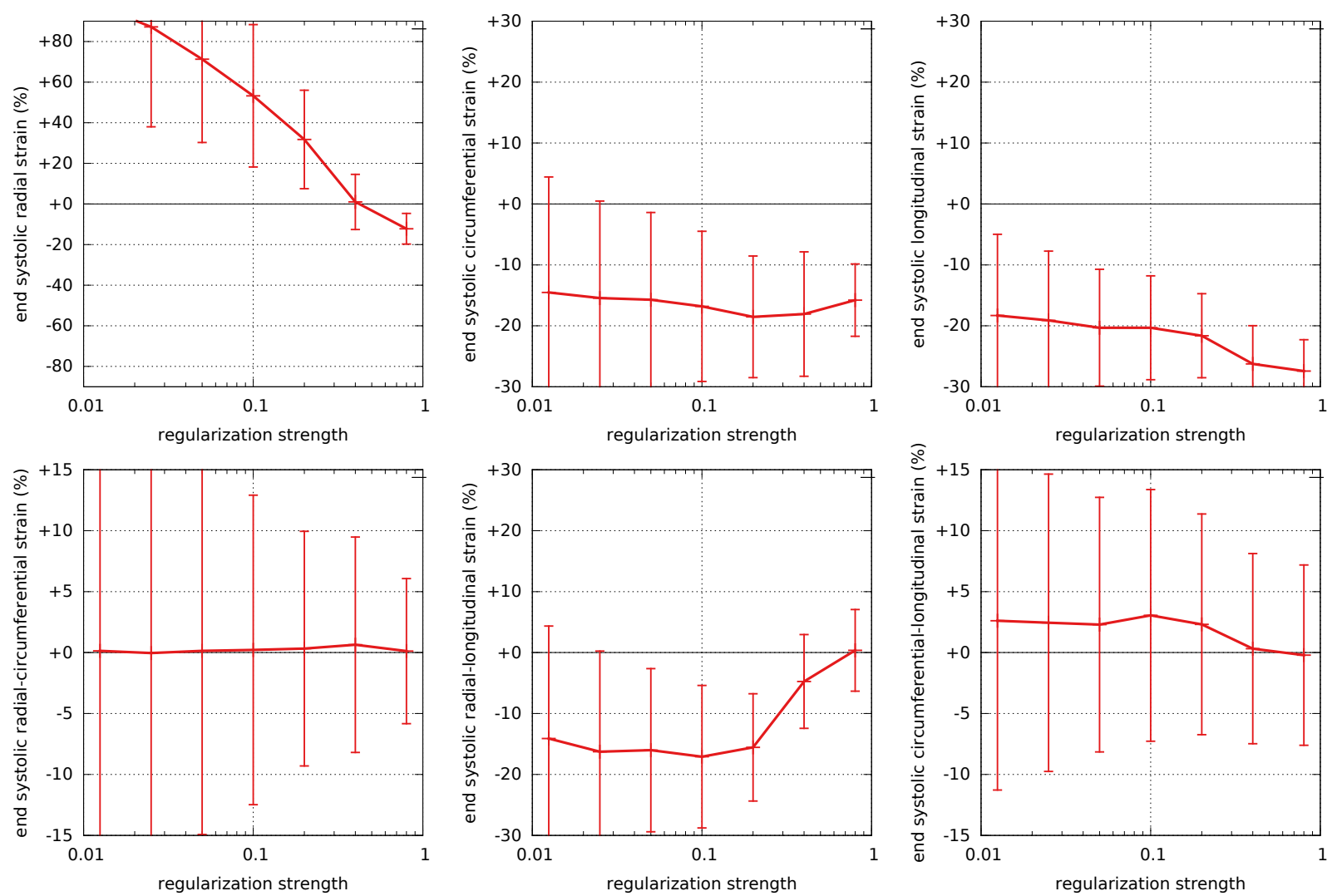

Figure 9: Results on in vivo untagged data. Green-Lagrange strain components at end-systole with respect to end-diastole (mean \pm standard deviation over the ventricle) as a function of regularization strength. As for tagged images, the equilibrium gap regularization has a limited impact on the registration. 
myocardial wall in the untagged images. This is induced by the motion of the neighboring features to the myocardium such as papillary muscles and pericardial fluid, which are present in the boundary layer of elements used to improve tracking of endocardium \& epicardium (see Section 2.2.3 for details). Indeed, tracking without boundary layer (not shown here) shows much reduced radial thickening, and almost no twist at all. Nevertheless, this is not a local measurement per se. We will further investigate the ability to measure heterogeneous strain fields, in case of myocardial infarction for instance.

\subsection{Limitations}

The proposed method has a number of limitations that we want to emphasize here. First of all, the finite element approach to image registration requires a segmentation and a mesh for the processing, which can be a drawback compared to other methods that work directly on the images. However, having a finite element mesh as support of the sought after displacement fields allows to define consistent functional spaces, with specific smoothness properties; moreover it opens the door for the combined processing of multiple images with different orientations and discretizations.

This points to another limitation of the current implementation of the method, where only one image set can be used at a time. Consequently, short-axis cine stacks need to be interpolated in the longitudinal direction, leading to registration errors as seen on Figure 10. Combining short-axis cine stacks and long-axis cine slices, and even untagged and tagged images, will probably improve registration significantly.

Specifically to the regularization method introduced here, even though equilibrium gap regularization was designed to have a limited bias on the strain results, it might still have a non-negligible impact on strains. Indeed, any mapping that is the solution of a hyperelastic body in equilibrium with surface loads is the minimizer of $\Psi^{\text {reg,equil }}$, so that the actual solution is probably close to a minimizer - this must be compared to hyperelastic regularization, where only the null mapping is a minimizer of the $\Psi^{\text {reg,hyper }}$, hence the large bias on strain results. However, because of anisotropy, contraction, and possibly non-affine deformation patterns, the actual solution might still be far from the equilibrium solution of the isotropic hyperelastic model postulated here, which could explain the poor tracking of radial strain found in Figure 7. It could also explain the fact that radial strains do not converge toward a given value when decreasing regularization strength, but keep increasing, on both tagged and untagged images, as seen in Figures $5 \&$ 9. More complex behavior laws could be used to address this issue.

\section{Conclusion}

Equilibrated warping is a powerful method for non-rigid registration of images involving arbitrary large deformation. The equilibrium gap constraint regularizes the image registration problem, even in presence of noise, with limited effect on strain measurement, as demonstrated using synthetic data. The method has been implemented based on FEniCS and VTK, providing an efficient tool for 2D \& 3D images registration. When applied to cardiac MR images, it allows to extract the main features of left ventricular deformation. 

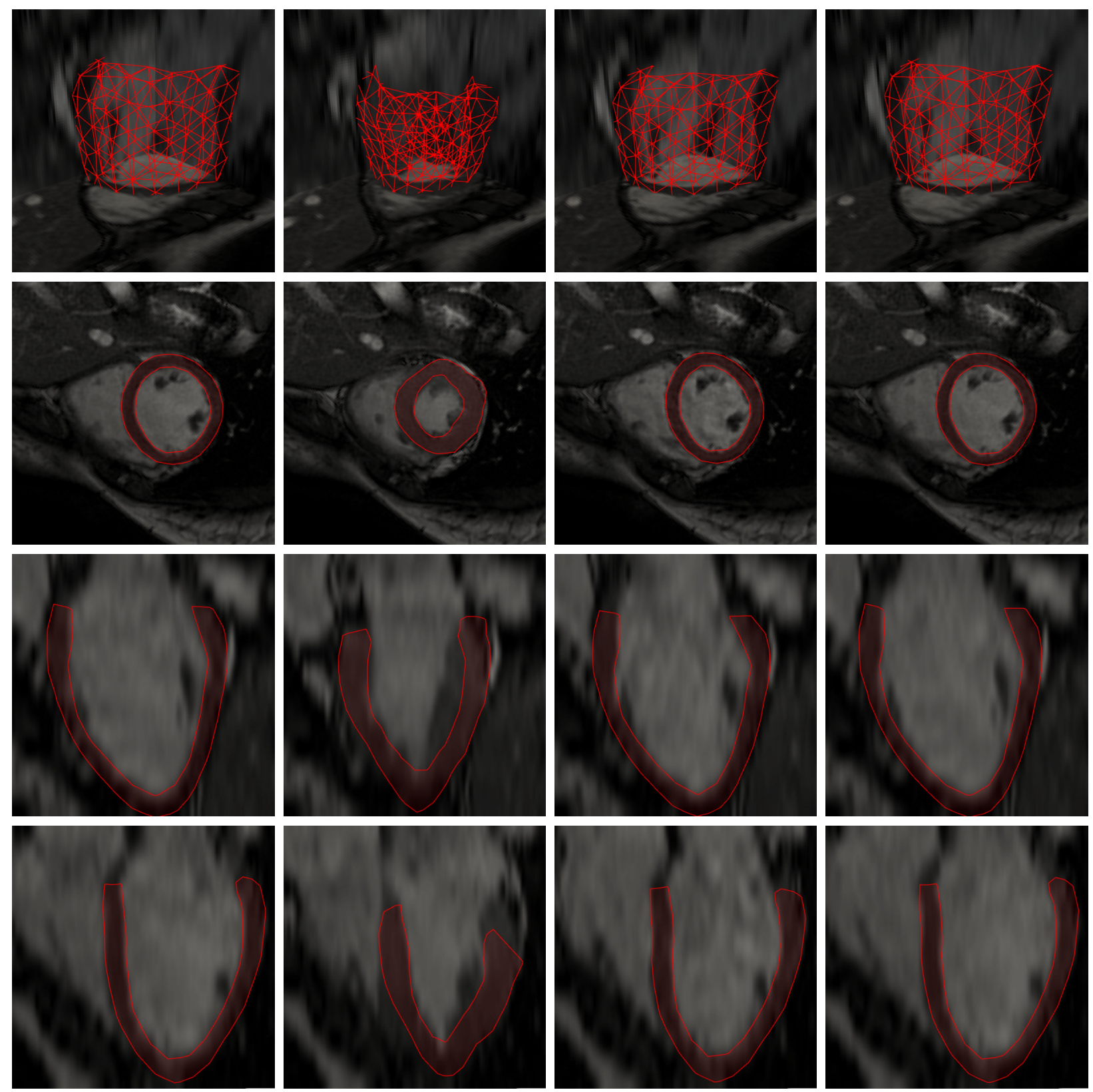

$$
t=0 \mathrm{~ms}
$$

$$
t=309 \mathrm{~ms}
$$

$$
t=618 m s
$$

$$
t=927 m s
$$

Figure 10: Results on in vivo untagged data. First row shows 3D view, while second, third and fourth rows show short axis, two-chamber and four-chamber views, respectively. 

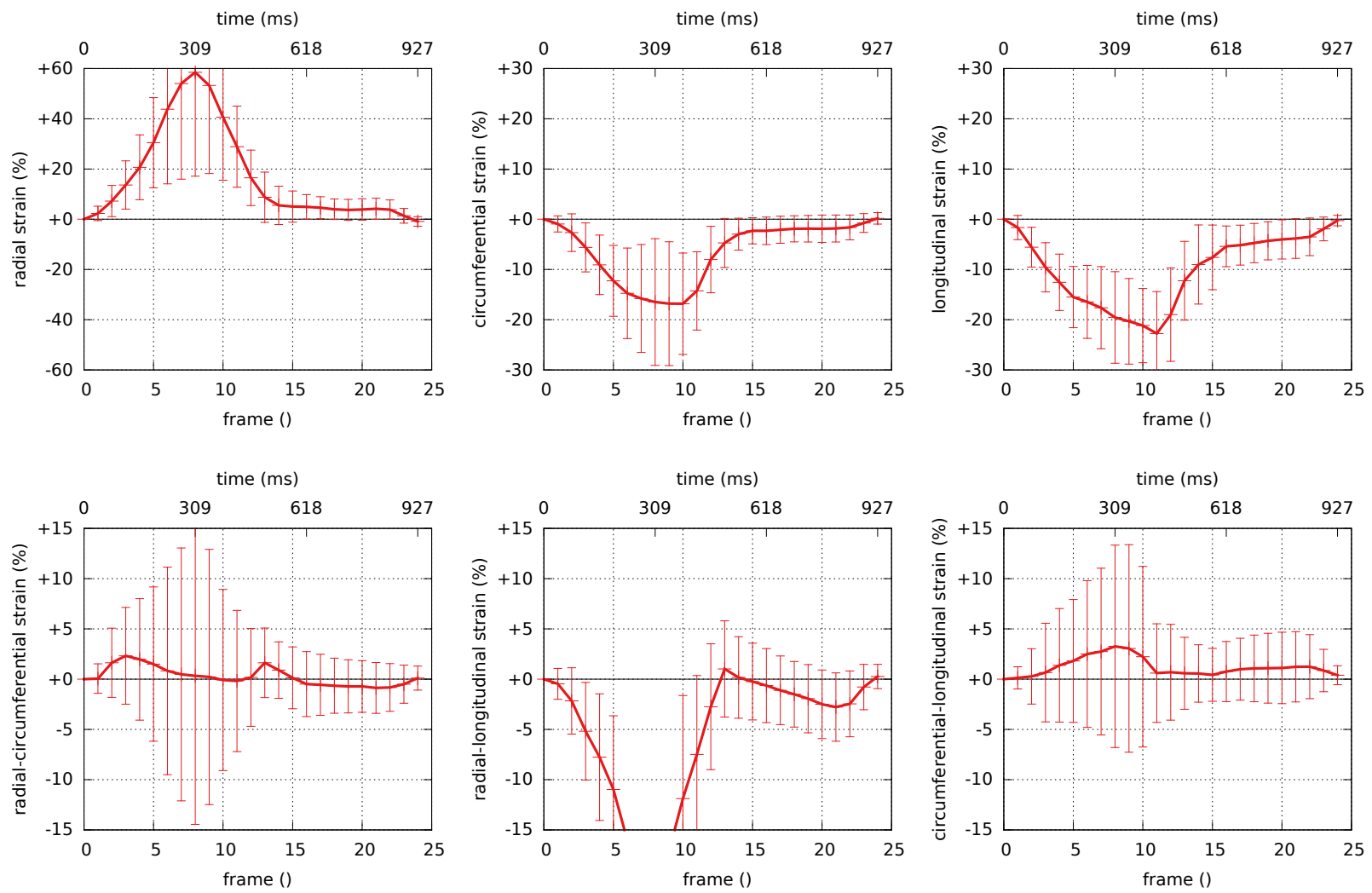

Figure 11: Results on in vivo untagged data. Green-Lagrange strain components with respect to enddiastole (mean \pm standard deviation over the ventricle) as a function of time, for a regularization strength of $\beta=0.1$. Despite a spurious radial-longitudinal motion, the method was able to capture circumferential and longitudinal shortenings, but also radial thickening as well as in-plane (i.e., radial-circumferential) and out-of-plane (i.e., circumferential-longitudinal) twists. 

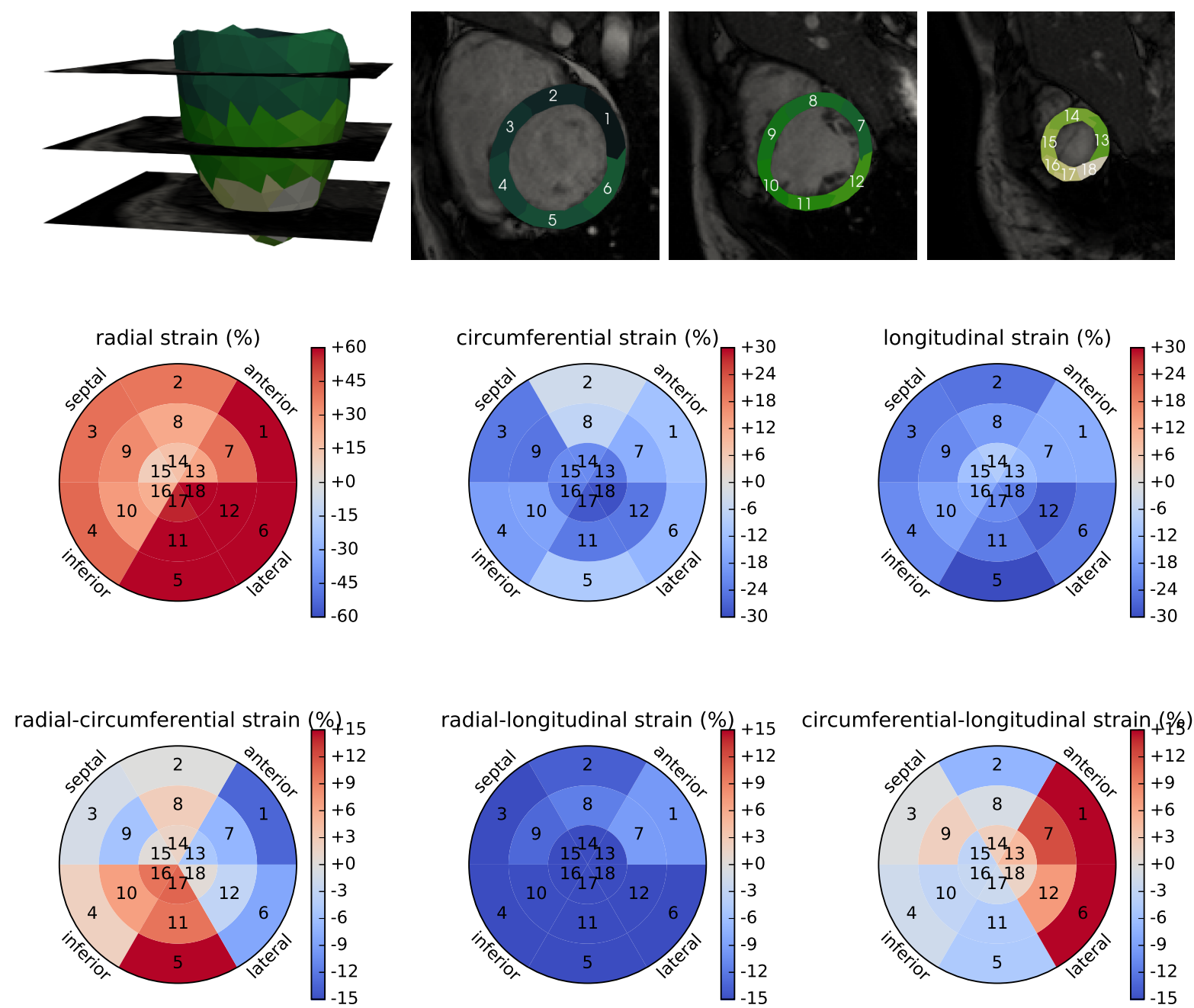

Figure 12: Results on in vivo untagged data. Top row: Left ventricular mesh split into 18 sectors (6 circumferential and 3 longitudinal sections). Bottom rows: Bull's eye plots of end-systolic Green-Lagrange strains, for a regularization strength of $\beta=0.1$. Numbers within sectors represent their ID, as described on the top row. Strains are generally more heterogeneous than for tagged images. However, it is interesting to note that radial strains are more important in the free wall than in the septum, which is expected since the septum is pressurized on both sides. 


\section{Appendix A. Validation using Motion Tracking Challenge}

In order to further validate the proposed image registration framework, we processed the public dataset of the Cardiac Motion Analysis Challenge that was held at the 2011 MICCAI workshop ${ }^{7}$, and is described in details in [Tobon-Gomez et al., 2013]. Briefly, the dataset consists of untagged (SSFP) and tagged (3DTAG) images (3DUS images were not processed), segmentations and markers that were manually tracked by experts on the images (i.e., ground truth, GT), for a dynamic phantom (PHANTOM) and fifteen healthy volunteers (V1, V2, V4-V16). Results, in terms of tracked markers, from the challenge competitors (INRIA, IUCL, MEVIS, UPF) are also provided.

We first generated volume meshes from the segmented surfaces using GMSH, as described in Section 2.2.2. (Segmented surface V8 was slightly distorted toward the base and could not be meshed, so this dataset was not analyzed.) Then, we run the equilibrated warping method on each dataset, with various regularization strengths, and interpolated the obtained displacement fields onto the markers. In order to assess the quality of the registration, the following normalized error on the markers trajectory was used:

$$
\text { err }=\frac{1}{n_{\text {markers }}} \sum_{m=1}^{n_{\text {markers }}} \frac{\sum_{f=1}^{n_{\text {frames }}}\left\|\underline{X}^{m}(f)-\underline{X}^{\mathrm{m}, \mathrm{GT}}(f)\right\|}{\sum_{f=1}^{n_{\text {frames }}-1}\left\|\underline{X}^{\mathrm{m}, \mathrm{GT}}(f+1)-\underline{X}^{\mathrm{m}, \mathrm{GT}}(f)\right\|},
$$

where $n_{\text {markers }}$ is the number of "valid" markers (i.e., markers that lie within the mesh in the reference configuration and can thus be tracked), $n_{\text {frames }}$ is the number of frames, $\underline{X}^{\mathrm{m}, \mathrm{GT}}(f)$ is the ground truth position of marker $m$ at frame $f$, and $\underline{X}^{\mathrm{m}}(f)$ is the tracked position of marker $m$ at frame $f$. In Figure A.1, we show this normalized markers error as a function of regularization strength, for the PHANTOM as well as the fourteen processed volunteers (V1, V2, V4-V7, V9-V16), for both 3DTAG and SSFP images. We also completed the normalized markers error for the results of the challenge competitors, which are shown on the plots. One can see that the regularization strength has a limited impact on registration. Moreover, the registration is overall quite satisfactory, with a normalized markers error always in the range of the other established methods. (With the exception of V11-SSFP results for $\beta=0.1$, for which the tracking failed.)

Figure A.2 shows the normalized markers error for all cases, for both 3DTAG and SSFP images, for all competitors as well as the proposed method for $\beta=0.1$ and best $\beta$ (which varies from one case to the other), in a bar plot. We also showed the average and standard deviation of normalized markers error over all cases (rightmost columns of the plot). This shows that the proposed method compares well to the other established methods.

\section{Appendix B. Vitae}

\section{Appendix B.1. Martin Genet}

Martin Genet obtained his PhD from École Normale Supérieure, Cachan, France, in 2010, and was then a postdoctoral fellow at the Lawrence Berkeley National Laboratory,

\footnotetext{
${ }^{7}$ http://www. cardiacatlas.org/challenges/motion-tracking-challenge
} 
California, USA, from 2010 to 2012. In 2012 he obtained a Marie-Curie International Outgoing fellowship to work on patient-specific cardiac modeling, at the University of California at San Francisco, USA, and Stanford University, Palo Alto, USA, from 2012 to 2014, and at the Swiss Federal Institute of Technology (ETH), Zurich, Switzerland, from 2014 to 2015. Since 2015 he is an Assistant Professor at École Polytechnique, Palaiseau, France, where he works on mechanical modeling and simulation, with data interaction, of soft tissues.

\section{Appendix B.2. Christian Stoeck}

Christian T. Stoeck obtained his PhD from ETH Zurich in Switzerland in 2014. He then worked as postdoctoral scholar at King's College London, UK, in 2014-2015 and at University of Zurich, Switzerland, in 2015-2017. The research of Christian Stoeck focuses on the development of novel cardiovascular imaging methodology for mechanical as well as microstructural imaging of the heart and its translation into pre-clinical as well as clinical environments.

\section{Appendix B.3. Constantin von Deuster}

Constantin von Deuster obtained his PhD in 2016 from King's College London, UK. He was working in a joint collaboration with the Division of Imaging Sciences and Biomedical Engineering at King's College London and the Institute for Biomedical Engineering at University and ETH Zurich, Switzerland. The focus of his thesis was on improving reliability of magnetic resonance diffusion imaging in the in vivo human heart. Since 2016 he is a postdoctoral fellow at the Institute for Biomedical Engineering at University and ETH Zurich, Switzerland, continuing his work on magnetic resonance diffusion imaging, including pulse sequence design and patient studies.

\section{Appendix B.4. Lik Chuan Lee}

Lik Chuan Lee is an Assistant Professor in the Department of Mechanical Engineering at Michigan State University. He received his $\mathrm{PhD}$ in Mechanical Engineering from the University of California, Berkeley, in 2010. He then worked as a postdoctoral scholar in the Department of Surgery at the University of California, San Francisco, between 2010 and 2014 prior to joining Michigan State University. Lik Chuan Lee's research interest is in computational mechanics and his current research focus is in computational cardiac modeling.

\section{Appendix B.5. Sebastian Kozerke}

Sebastian Kozerke, is a Professor of Biomedical Imaging at the Institute for Biomedical Engineering of the University and ETH Zurich, Switzerland, where he leads the Cardiovascular Magnetic Resonance research. He obtained his PhD degree and the Venia legendi from ETH Zurich in 2000 and 2005, respectively. In 2002-03 he was a research associate at the Division of Imaging Sciences at King's College London, UK. He was elected as a Professor and Chair of MR physics at King's College London in 2008 before being promoted to Professor at the University of Zurich in 2010. In 2014 he was elected as full Professor at ETH Zurich. The research of Sebastian Kozerke spans from developments in MR pulse sequence 
design, scan acceleration techniques, novel information encoding concepts, dynamic nuclear polarization techniques to experimental validation and clinical translation of MR methods.

\section{References}

Alnæs, M., Blechta, J., Hake, J., Johansson, A., Kehlet, B., Logg, A., Richardson, C., Ring, J., Rognes, M. E., Wells, G. N., 2015. The FEniCS Project Version 1.5. Tech. rep., University Library Heidelberg.

Arts, T., Prinzen, F. W., Delhaas, T., Milles, J. R., Rossi, A. C., Clarysse, P., 2010. Mapping displacement and deformation of the heart with local sine-wave modeling. IEEE Transactions on Medical Imaging $29(5), 1114-23$

Axel, L., Dougherty, L., 1989. MR imaging of motion with spatial modulation of magnetization. Radiology $171,841-845$.

Bertini, M., Delgado, V., Nucifora, G., Ajmone Marsan, N., Ng, A. C. T., Shanks, M., Antoni, M. L., van de Veire, N. R. L., van Bommel, R. J., Rapezzi, C., Schalij, M. J., Bax, J. J., Nov. 2010. Left ventricular rotational mechanics in patients with coronary artery disease: Differences in subendocardial and subepicardial layers. Heart 96 (21), 1737-1743.

Burger, M., Modersitzki, J., Ruthotto, L., Jan. 2013. A Hyperelastic Regularization Energy for Image Registration. SIAM Journal on Scientific Computing 35 (1), B132-B148.

Chapelle, D., Fragu, M., Mallet, V., Moireau, P., 2013. Fundamental principles of data assimilation underlying the Verdandi library: Applications to biophysical model personalization within euHeart. Medical and Biological Engineering and Computing 51, 1221-1233.

Christensen, G. E., Rabbitt, R. D., Miller, M. I., Jan. 1996. Deformable templates using large deformation kinematics. IEEE transactions on image processing : a publication of the IEEE Signal Processing Society 5 (10), 1435-47.

Ciarlet, P. G., Geymonat, G., 1982. Sur les lois de comportement en élasticité non-linéaire compressible. Comptes Rendus de l'Académie des Sciences Série II 295, 423-426.

Claire, D., Hild, F., Roux, S., 2004. A finite element formulation to identify damage fields: The equilibrium gap method. International Journal for Numerical Methods in Engineering 61 (2), 189-208.

Delingette, H., Billet, F., Wong, K. C. L., Sermesant, M., Rhode, K., Ginks, M., Rinaldi, C. A., Razavi, R., Ayache, N., Jan. 2012. Personalization of Cardiac Motion and Contractility From Images Using Variational Data Assimilation. IEEE Transactions on Biomedical Engineering 59 (1), 20-24.

Fischer, S. E., McKinnon, G. C., Maier, S. E., Boesiger, P., Aug. 1993. Improved myocardial tagging contrast. Magnetic Resonance in Medicine 30 (2), 191-200.

Garot, J., Bluemke, D. A., Osman, N. F., Rochitte, C. E., McVeigh, E. R., Zerhouni, E. A., Prince, J. L., Lima, J. A. C., Mar. 2000. Fast Determination of Regional Myocardial Strain Fields From Tagged Cardiac Images Using Harmonic Phase MRI. Circulation 101 (9), 981-988.

Genet, M., Lee, L. C., Ge, L., Acevedo-Bolton, G., Jeung, N., Martin, A. J., Cambronero, N., Boyle, A. J., Yeghiazarians, Y., Kozerke, S., Guccione, J. M., Aug. 2015. A Novel Method for Quantifying Smooth Regional Variations in Myocardial Contractility Within an Infarcted Human Left Ventricle Based on Delay-Enhanced Magnetic Resonance Imaging. Journal of Biomechanical Engineering 137 (8).

Genet, M., Lee, L. C., Nguyen, R., Haraldsson, H., Acevedo-Bolton, G., Zhang, Z., Ge, L., Ordovas, K., Kozerke, S., Guccione, J. M., 2014. Distribution of normal human left ventricular myofiber stress at end diastole and end systole: A target for in silico design of heart failure treatments. Journal of Applied Physiology 117, 142-52.

Genet, M., Stoeck, C. T., von Deuster, C., Lee, L. C., Guccione, J. M., Kozerke, S., 2016. Finite Element Digital Image Correlation for Cardiac Strain Analysis from 3D Whole-Heart Tagging. In: 24th Annual Meeting of the International Society for Magnetic Resonance in Medicine (ISMRM2016).

Geuzaine, C., Remacle, J.-F., Sep. 2009. Gmsh: A three-dimensional finite element mesh generator with built-in pre- and post-processing facilities. International Journal for Numerical Methods in Engineering 79 (11), 1309-1331. 
Hild, F., Bouterf, A., Chamoin, L., Leclerc, H., Mathieu, F., Neggers, J., Pled, F., Tomičević, Z., Roux, S., Dec. 2016. Toward 4D mechanical correlation. Advanced Modeling and Simulation in Engineering Sciences $3(1)$.

Hild, F., Roux, S., May 2006. Digital Image Correlation: From Displacement Measurement to Identification of Elastic Properties - a Review. Strain 42 (2), 69-80.

Hild, F., Roux, S., Nov. 2012. Comparison of Local and Global Approaches to Digital Image Correlation. Experimental Mechanics 52 (9), 1503-1519.

Holzapfel, G. A., 2000. Nonlinear Solid Mechanics: A Continuum Approach for Engineering. Wiley, Chichester.

Ibrahim, E.-S. H., Jan. 2011. Myocardial tagging by cardiovascular magnetic resonance: Evolution of techniques-pulse sequences, analysis algorithms, and applications. Journal of cardiovascular magnetic resonance : official journal of the Society for Cardiovascular Magnetic Resonance 13 (1), 36.

Imperiale, A., Chabiniok, R., Moireau, P., Chapelle, D., 2011. Constitutive parameter estimation methodology using tagged-MRI data. In: Lecture Notes in Computer Science. Vol. 6666 LNCS. pp. 409-417.

Krishnamurthy, A., Villongco, C. T., Chuang, J., Frank, L. R., Nigam, V., Belezzuoli, E., Stark, P., Krummen, D. E., Narayan, S. M., Omens, J. H., McCulloch, A. D., Kerckhoffs, R. C. P., Jul. 2013. PatientSpecific Models of Cardiac Biomechanics. Journal of computational physics 244, 4-21.

Le Tallec, P., 1994. Numerical Methods for Nonlinear Elasticity. In: Handbook of Numerical Analysis. Vol. 3. pp. $465-622$.

Leclerc, H., Périé, J.-N., Roux, S., Hild, F., 2010. Voxel-Scale Digital Volume Correlation. Experimental Mechanics 51 (4), 479-490.

Lee, L. C., Genet, M., Dang, A. B., Ge, L., Guccione, J. M., Ratcliffe, M. B., May 2014. Applications of computational modeling in cardiac surgery. Journal of Cardiac Surgery 29 (3), 293-302.

Lenoir, N., Bornert, M., Desrues, J., Bésuelle, P., Viggiani, G., Aug. 2007. Volumetric Digital Image Correlation Applied to X-ray Microtomography Images from Triaxial Compression Tests on Argillaceous Rock. Strain 43 (3), 193-205.

Logg, A., Mardal, K.-A., Wells, G. (Eds.), 2012. Automated Solution of Differential Equations by the Finite Element Method: The FEniCS Book. No. 84 in Lecture Notes in Computational Science and Engineering. Springer, Heidelberg.

Mansi, T., Pennec, X., Sermesant, M., Delingette, H., Ayache, N., Mar. 2011. iLogDemons: A Demons-Based Registration Algorithm for Tracking Incompressible Elastic Biological Tissues. International Journal of Computer Vision 92 (1), 92-111.

May-Newman, K., Omens, J. H., Pavelec, R. S., McCulloch, A. D., 1994. Three-dimensional transmural mechanical interaction between the coronary vasculature and passive myocardium in the dog. Circulation Research 74 (6), 1166-1178.

McLeod, K., Prakosa, A., Mansi, T., Sermesant, M., Pennec, X., 2012. An Incompressible Log-Domain Demons Algorithm for Tracking Heart Tissue. In: Statistical Atlases and Computational Models of the Heart - Imaging and Modelling Challenges. pp. 55-67.

Moireau, P., Chapelle, D., Le Tallec, P., Jan. 2008. Joint state and parameter estimation for distributed mechanical systems. Computer Methods in Applied Mechanics and Engineering 197 (6-8), 659-677.

Moore, C. C., Lugo-Olivieri, C. H., McVeigh, E. R., Zerhouni, E. A., Feb. 2000. Three-dimensional systolic strain patterns in the normal human left ventricle: Characterization with tagged MR imaging. Radiology $214(2), 453-466$.

Osman, N. F., Kerwin, W. S., McVeigh, E. R., Prince, J. L., Dec. 1999. Cardiac motion tracking using CINE harmonic phase (HARP) magnetic resonance imaging. Magnetic Resonance in Medicine 42 (6), $1048-60$.

Passieux, J.-C., Périé, J.-N., Nov. 2012. High resolution digital image correlation using proper generalized decomposition: PGD-DIC. International Journal for Numerical Methods in Engineering 92 (6), 531-550.

Phatak, N. S., Maas, S. A., Veress, A. I., Pack, N. A., Di Bella, E. V. R., Weiss, J. A., Apr. 2009. Strain measurement in the left ventricle during systole with deformable image registration. Medical image analysis $13(2), 354-61$.

Pierré, J.-E., Passieux, J.-C., Périé, J.-N., Bugarin, F., Robert, L., Feb. 2016. Unstructured finite element- 
based digital image correlation with enhanced management of quadrature and lens distortions. Optics and Lasers in Engineering 77, 44-53.

Press, W. H., Teukolsky, S. A., Vetterling, W. T., Flannery, B. P., 2007. Numerical Recipes: The Art of Scientific Computing, 3rd Edition. Cambridge University Press, Cambridge, UK ; New York.

Remacle, J.-F., Geuzaine, C., Compère, G., Marchandise, E., 2010. High-quality surface remeshing using harmonic maps. International Journal for Numerical Methods in Engineering.

Rutz, A. K., Ryf, S., Plein, S., Boesiger, P., Kozerke, S., Apr. 2008. Accelerated whole-heart 3D CSPAMM for myocardial motion quantification. Magnetic resonance in medicine 59 (4), 755-63.

Ryf, S., Spiegel, M. A., Gerber, M., Boesiger, P., Sep. 2002. Myocardial tagging with 3D-CSPAMM. Journal of Magnetic Resonance Imaging 16 (3), 320-325.

Sainte-Marie, J., Chapelle, D., Cimrman, R., Sorine, M., Nov. 2006. Modeling and estimation of the cardiac electromechanical activity. Computers \& Structures 84 (28), 1743-1759.

Schroeder, W., Martin, K., Lorensen, B., 2006. The Visualization Toolkit: An Object-Oriented Approach to 3D Graphics, 4th Edition. Kitware, Inc, Clifton Park, NY.

Sermesant, M., Moireau, P., Camara, O., Sainte-Marie, J., Andriantsimiavona, R., Cimrman, R., Hill, D. L. G., Chapelle, D., Razavi, R., 2006. Cardiac function estimation from MRI using a heart model and data assimilation: Advances and difficulties. Medical Image Analysis 10, 642-656.

Shehata, M. L., Cheng, S., Osman, N. F., Bluemke, D. A., Lima, J. A., 2009. Myocardial tissue tagging with cardiovascular magnetic resonance. Journal of Cardiovascular Magnetic Resonance 11 (1), 55.

Sotiras, A., Davatzikos, C., Paragios, N., Jul. 2013. Deformable Medical Image Registration: A Survey. IEEE Transactions on Medical Imaging 32 (7), 1153-1190.

Stoeck, C. T., Manka, R., Boesiger, P., Kozerke, S., 2012. Undersampled Cine 3D tagging for rapid assessment of cardiac motion. Journal of Cardiovascular Magnetic Resonance 14 (1), 60.

Stoeck, C. T., von Deuster, C., Genet, M., Atkinson, D., Kozerke, S., 2015. Second-order motioncompensated spin echo diffusion tensor imaging of the human heart. Magnetic Resonance in Medicine.

Sutton, M. A., Hild, F., Jan. 2015. Recent Advances and Perspectives in Digital Image Correlation. Experimental Mechanics 55 (1), 1-8.

Thirion, J.-P., Sep. 1998. Image matching as a diffusion process: An analogy with Maxwell's demons. Medical Image Analysis 2 (3), 243-260.

Tobon-Gomez, C., De Craene, M., McLeod, K., Tautz, L., Shi, W., Hennemuth, A., Prakosa, A., Wang, H., Carr-White, G. S., Kapetanakis, S., Lutz, A., Rasche, V., Schaeffter, T., Butakoff, C., Friman, O., Mansi, T., Sermesant, M., Zhuang, X., Ourselin, S., Peitgen, H.-O., Pennec, X., Razavi, R., Rueckert, D., Frangi, A. F., Rhode, K. S., Aug. 2013. Benchmarking framework for myocardial tracking and deformation algorithms: An open access database. Medical Image Analysis 17 (6), 632-648.

Vercauteren, T., Pennec, X., Perchant, A., Ayache, N., 2008. Symmetric Log-Domain Diffeomorphic Registration: A Demons-Based Approach. In: Metaxas, D., Axel, L., Fichtinger, G., Székely, G. (Eds.), Medical Image Computing and Computer-Assisted Intervention — MICCAI 2008. Vol. 5241. Springer Berlin Heidelberg, Berlin, Heidelberg, pp. 754-761.

Veress, A. I., Gullberg, G. T., Weiss, J. A., Jul. 2005. Measurement of Strain in the Left Ventricle during Diastole with cine-MRI and Deformable Image Registration. Journal of Biomechanical Engineering 127 (7), 1195.

von Deuster, C., Stoeck, C. T., Genet, M., Atkinson, D., Kozerke, S., 2015. Spin echo versus stimulated echo diffusion tensor imaging of the in vivo human heart. Magnetic Resonance in Medicine.

Wang, H., Amini, A. A., 2012. Cardiac motion and deformation recovery from MRI: A review. IEEE transactions on medical imaging 31 (2), 487-503.

Wang, H., Stoeck, C. T., Kozerke, S., Amini, A. A., Jan. 2013. Analysis of 3D cardiac deformations with 3D SinMod. Annual International Conference of the IEEE Engineering in Medicine and Biology Society 2013, 4386-9.

Zerhouni, E. A., Parish, D. M., Rogers, W. J., Yang, A., Shapiro, E. P., Oct. 1988. Human heart: Tagging with MR imaging-a method for noninvasive assessment of myocardial motion. Radiology 169 (1), 59-63. 
3DTAG
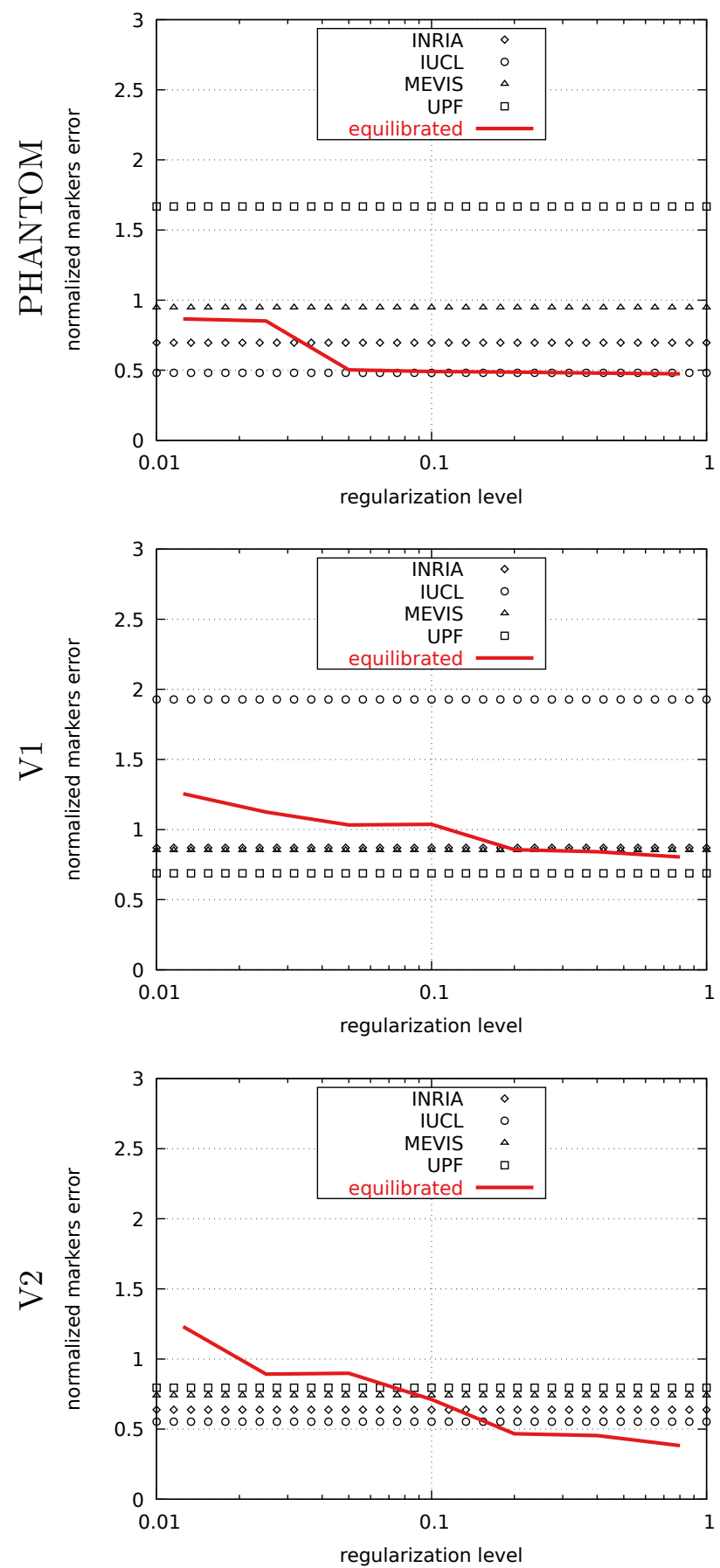

SSFP
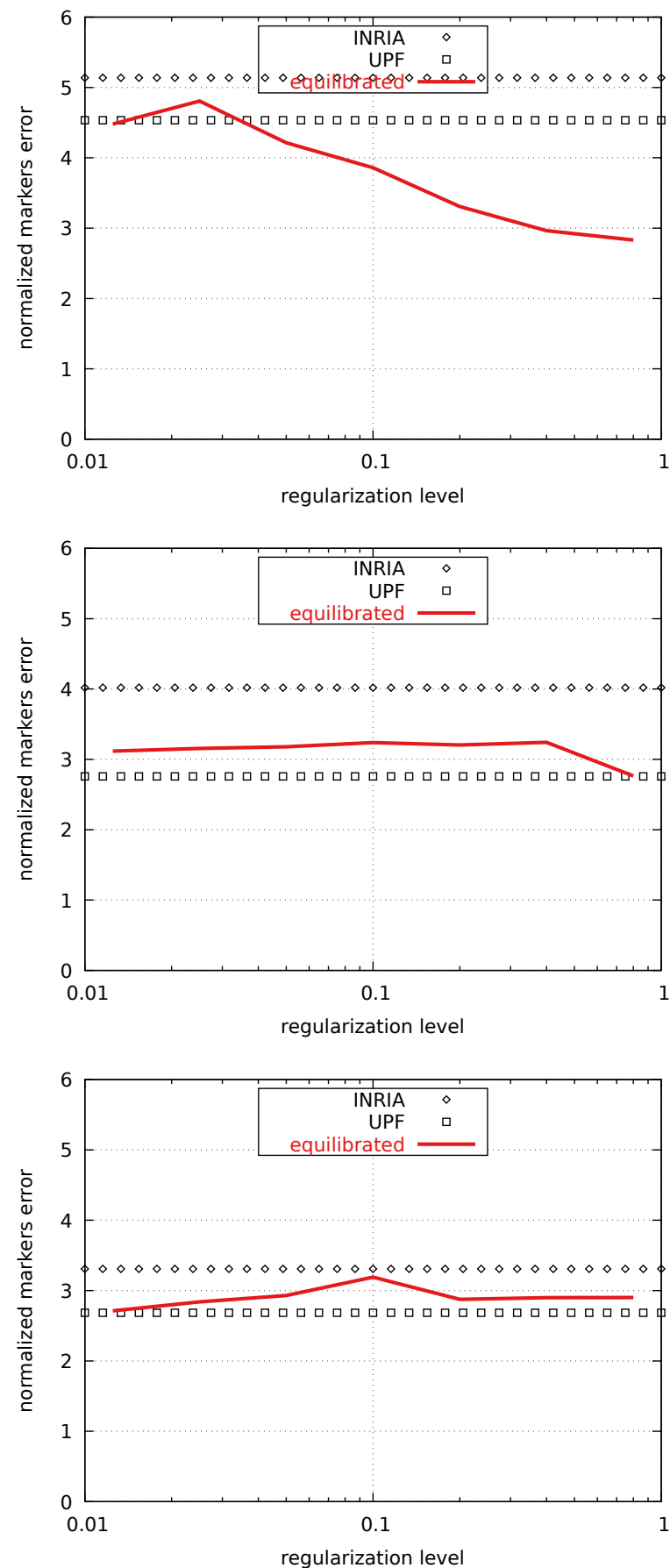

Figure A.1: Validation using Motion Tracking Challenge. Normalized markers error as a function of regularization strength, compared to results obtained by challengers, for 3DTAG \& SSFP images, for all cases. One can see that the equilibrium gap regularization is robust, and that the registration is not critically dependent on regularization strength, which can then be roughly determined. 
3DTAG
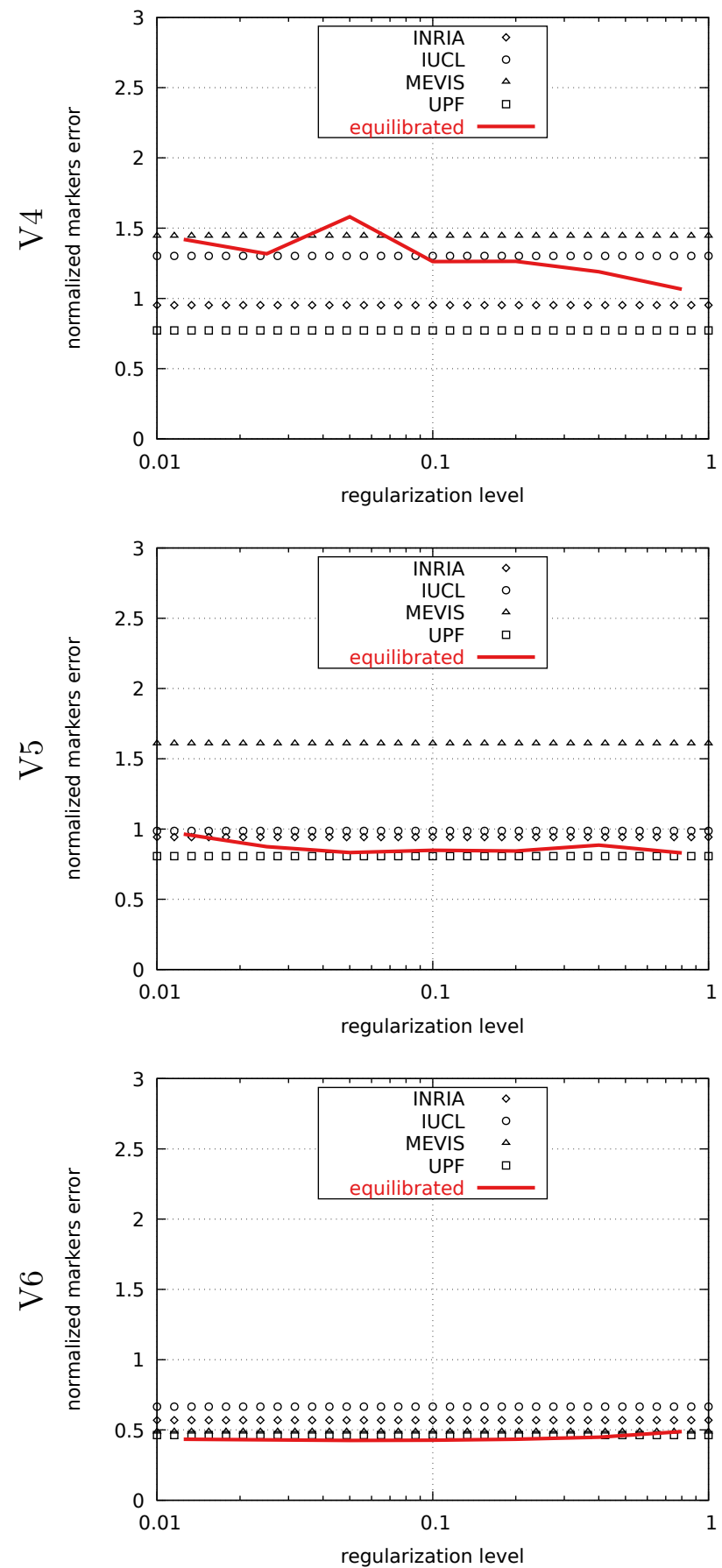

SSFP
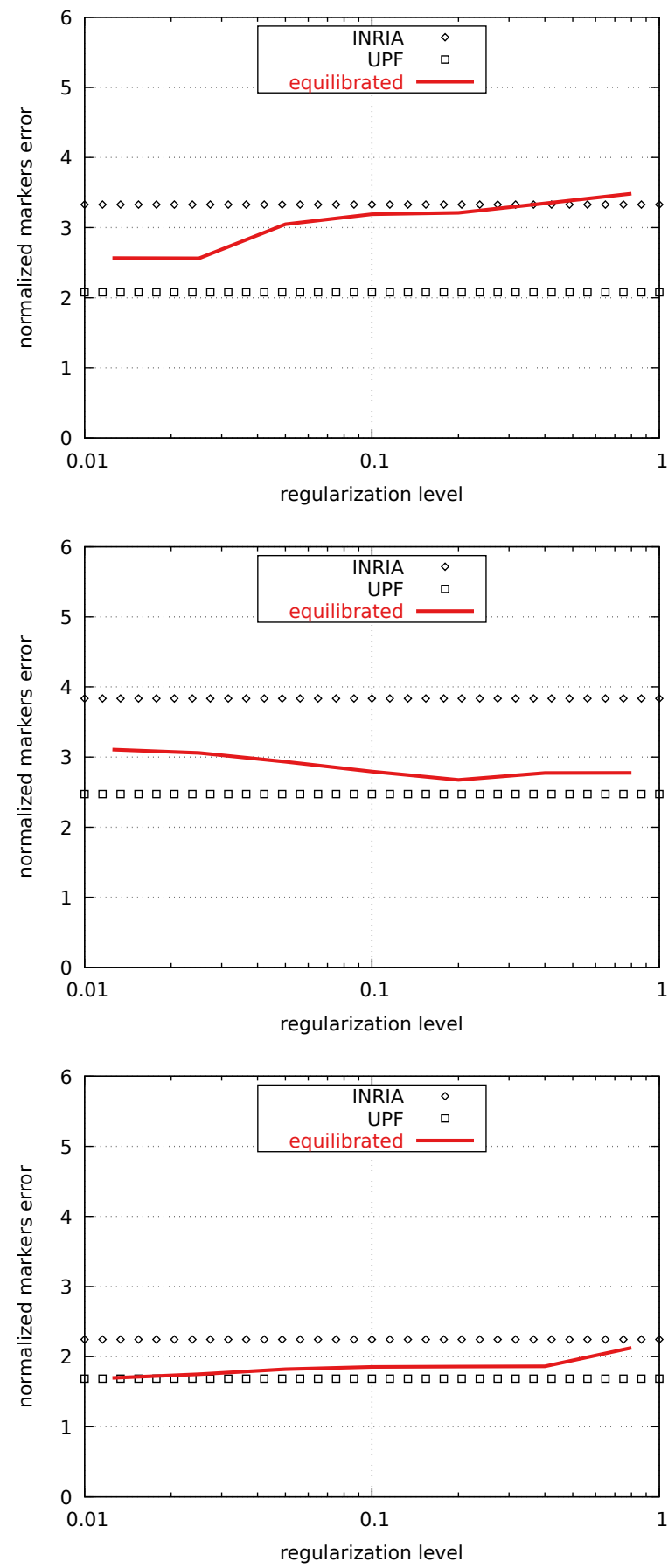

Figure A.1: (Cont.) 
3DTAG
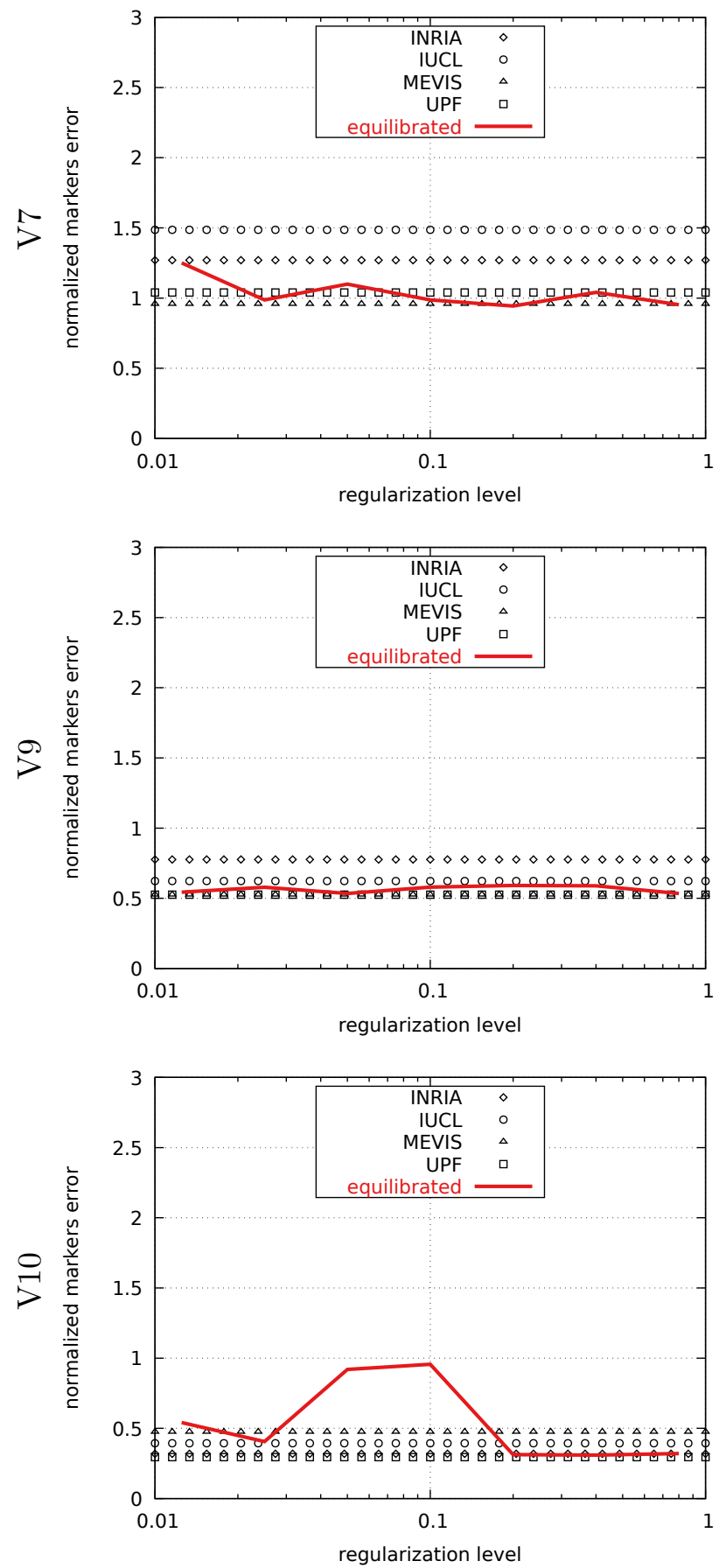

SSFP
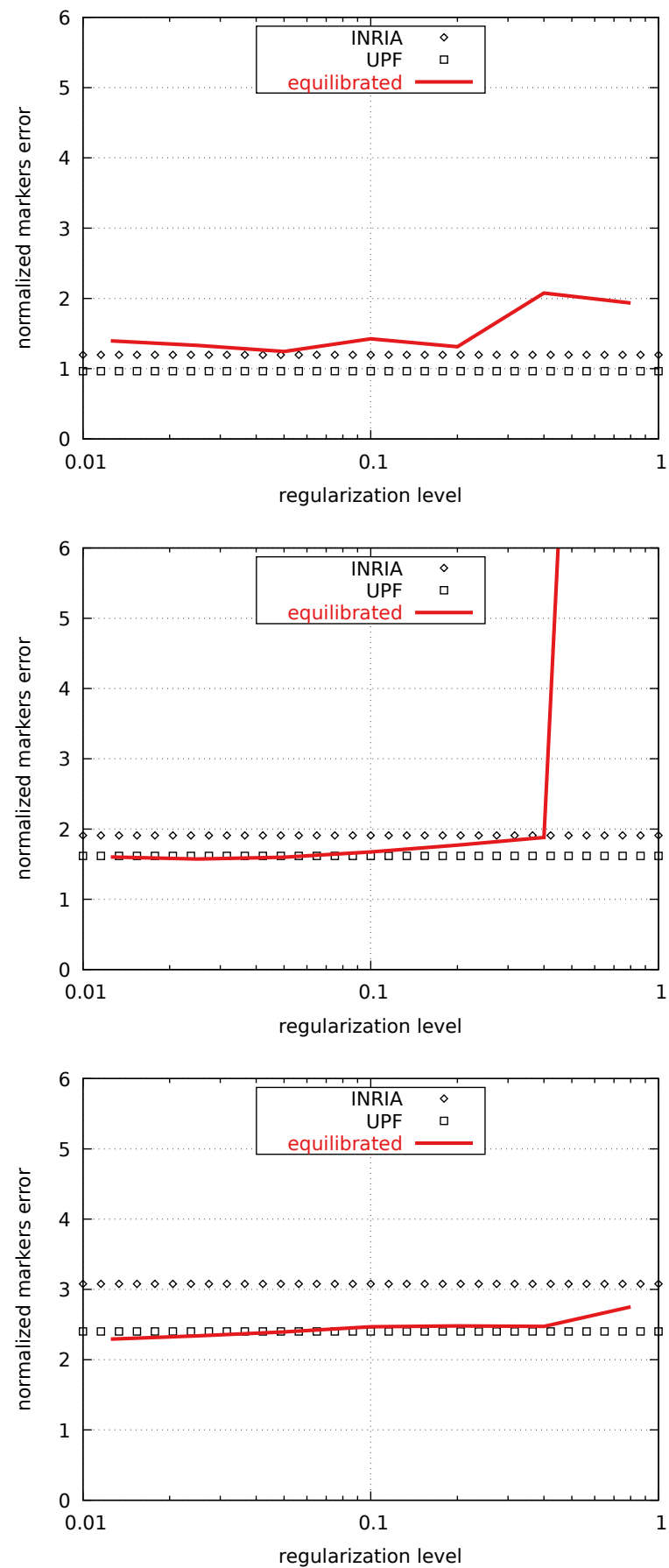

Figure A.1: (Cont.) 
3DTAG
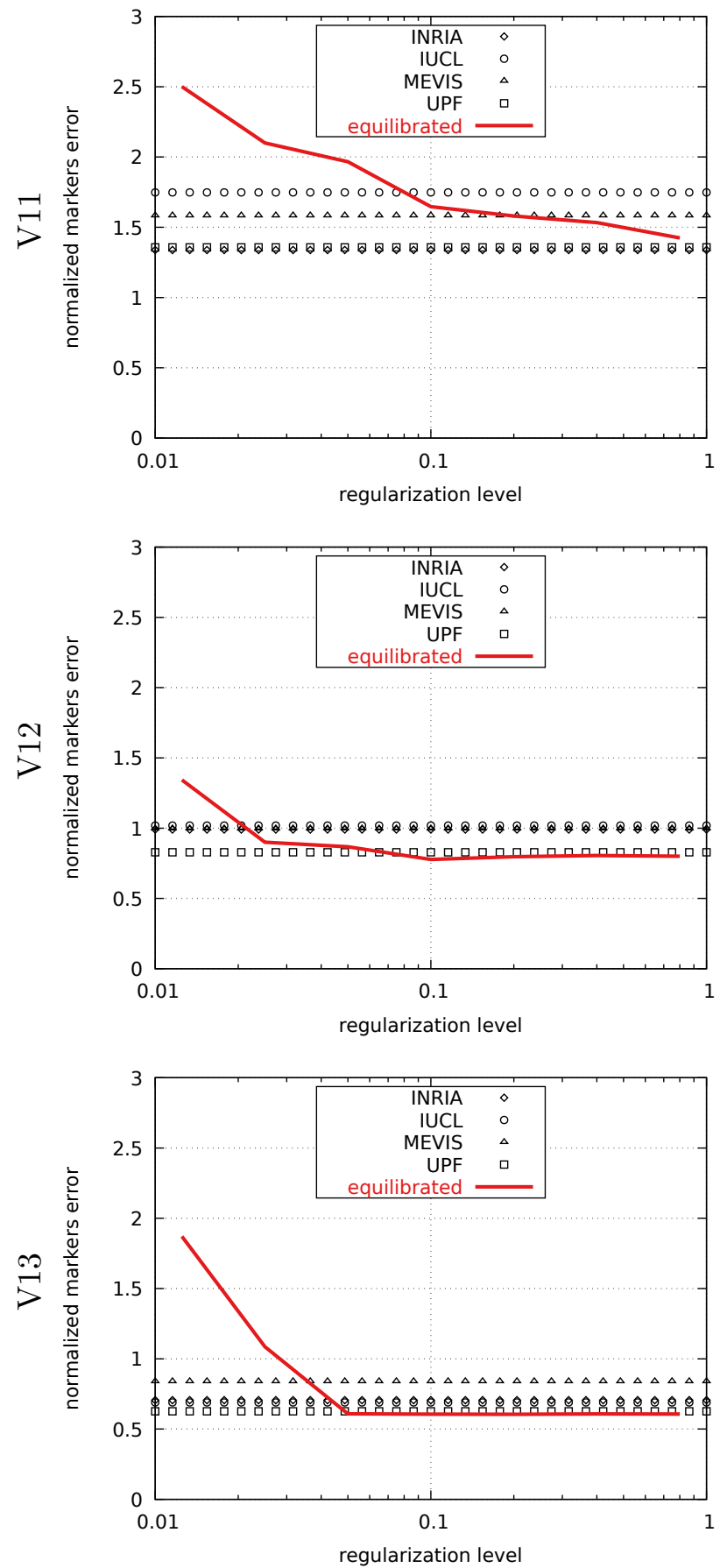

SSFP
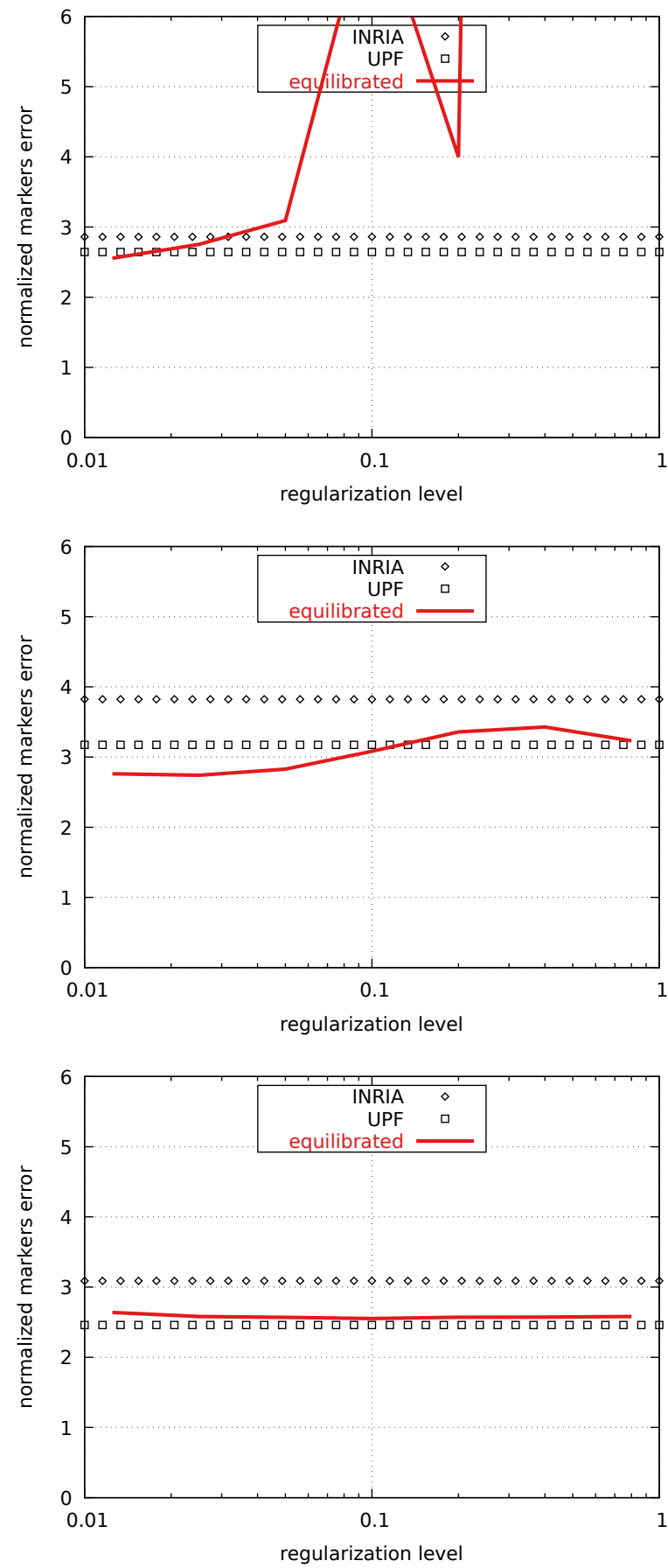

Figure A.1: (Cont.) 
3DTAG


SSFP
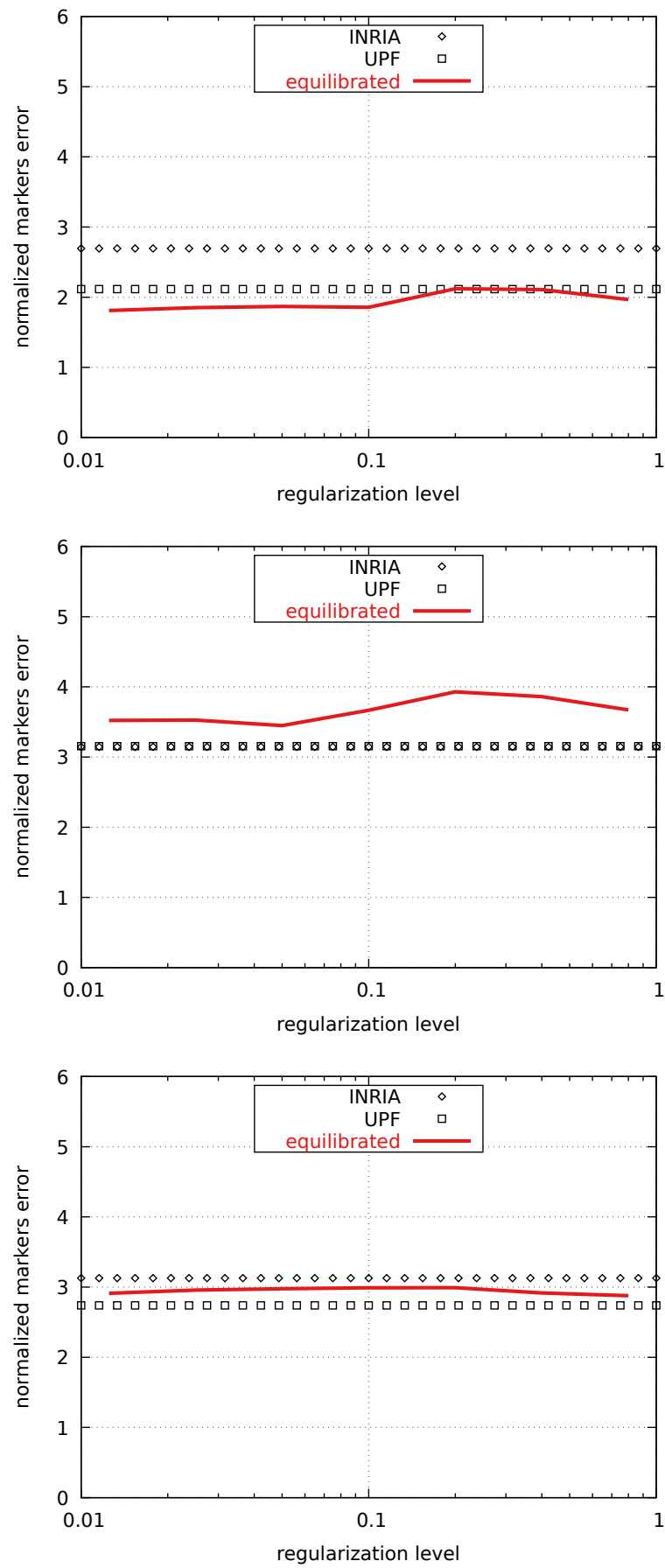

Figure A.1: (Cont.) 

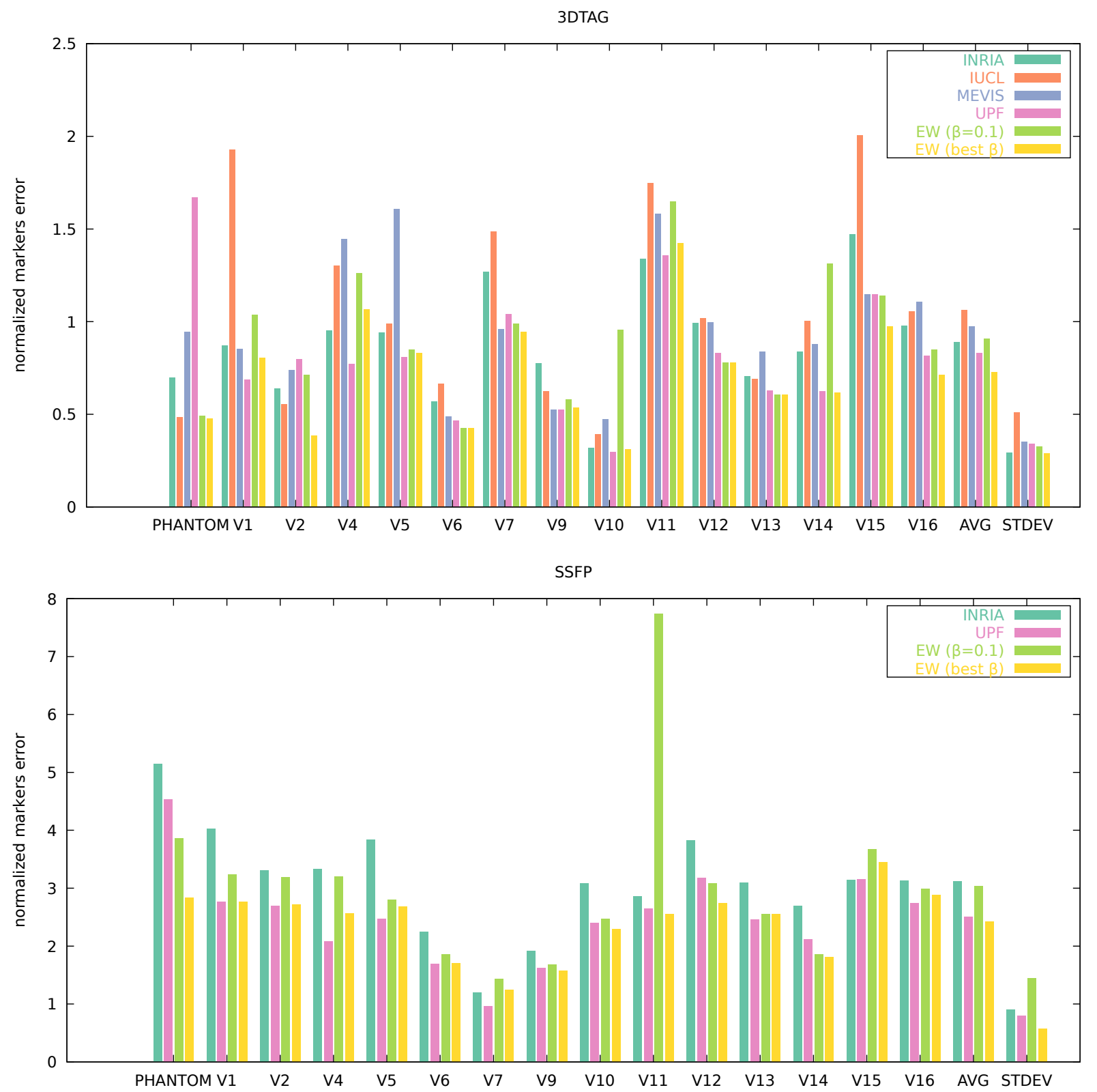

Figure A.2: Validation using Motion Tracking Challenge. Normalized markers error, for both 3DTAG \& SSFP images and for all cases, as well as normalized markers error mean and standard deviation over all cases. One can see that the proposed method compares well to the other established registration methods. 